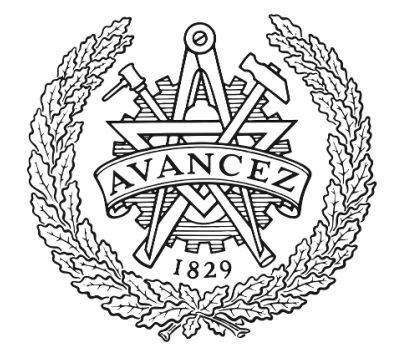

CHALMERS

UNIVERSITY OF TECHNOLOGY

\title{
Limited Feedforward Waveform Design for OFDM Dual-Functional Radar-Communications
}

Downloaded from: https://research.chalmers.se, 2023-04-26 10:37 UTC

Citation for the original published paper (version of record):

Keskin, F., Koivunen, V., Wymeersch, H. (2021). Limited Feedforward Waveform Design for OFDM Dual-Functional Radar-Communications. IEEE Transactions on Signal Processing, 69: 2955-2970. http://dx.doi.org/10.1109/TSP.2021.3076894

N.B. When citing this work, cite the original published paper.

C2021 IEEE. Personal use of this material is permitted.

However, permission to reprint/republish this material for advertising or promotional purposes 


\title{
Limited Feedforward Waveform Design for OFDM Dual-Functional Radar-Communications
}

\author{
Musa Furkan Keskin, Member, IEEE, Visa Koivunen, Fellow, IEEE, and Henk Wymeersch, Senior Member, IEEE
}

\begin{abstract}
We consider the problem of time-frequency waveform design for an OFDM dual-functional radar-communications (DFRC) system that communicates with an OFDM receiver while simultaneously estimating target parameters using the backscattered signals. In particular, the goal is to achieve a favorable performance trade-off between radar and communications by optimizing subcarrier powers in a time-frequency region of interest. First, we focus on radar-optimal waveform design to minimize the Cramér-Rao bound (CRB) on delay-Doppler estimation subject to an integrated side-lobe level (ISL) constraint in the delayDoppler ambiguity domain, where a convex-concave procedure (CCP) is devised to solve the resulting non-convex fractional program. Next, we investigate the problem of DFRC trade-off waveform design to optimize the communications rate under radar similarity constraint. Unlike the traditional DFRC systems which ignore feedforward overhead for conveying transmit waveform control information, we assume the existence of a lowrate feedforward channel between the DFRC transceiver and the communications receiver. Relying on the covariance matrix of linear minimum mean-squared-error (LMMSE) estimates of input symbols, we derive a novel communications metric as a function of both subcarrier powers and forwarded control information, and propose a joint waveform and control signaling optimization (JWCSO) strategy that leverages the sparsity and rank-one structure of DFRC waveforms within an alternating maximization framework. Simulation results show that the proposed JWCSO approach provides significant performance gains over the conventional feedforward-agnostic waveforms and achieves nearoptimal radar-communications trade-off performance, reaching the boundary of the CRB-capacity region with only a limited feedforward information.
\end{abstract}

Index Terms- OFDM, dual-functional radar-communications, waveform design, limited feedforward, delay-Doppler ambiguity.

\section{INTRODUCTION}

\section{A. Background and Motivation}

Joint radar-communications (JRC) systems have recently gathered a great deal of interest as spectral congestion becomes a compelling issue in $5 \mathrm{G}$ and beyond wireless networks [1] [6]. As one of the dominant threads in JRC research, dualfunctional radar-communications (DFRC) system design aims to develop a single hardware platform that can perform radar sensing and communications simultaneously [2], [7], [8]. The essential merit of DFRC approach over separate radar and communications systems lies in its ability to provide spectrum efficiency and hardware reuse that helps reduce cost, size and power consumption [9], [10]. In vehicular applications, dual-functionality can be accomplished by exploiting the existing vehicle-to-vehicle (V2V) communications standards (e.g.,

Musa Furkan Keskin and Henk Wymeersch are with the Department of Electrical Engineering, Chalmers University of Technology, SE 41296 Gothenburg, Sweden (e-mail: furkan@chalmers.se). Visa Koivunen is with the Department of Signal Processing and Acoustics, Aalto University, FI 00076 Aalto, Finland. This work is supported, in part, by Vinnova gran 2018-01929, MSCA-IF grant 888913 (OTFS-RADCOM) and the European Commission through the H2020 project Hexa-X (Grant Agreement no. 101015956). This paper has supplementary downloadable material available at http://ieeexplore.ieee.org., provided by the author. The material includes the proofs and derivations. This material is $823 \mathrm{~KB}$ in size.
IEEE 802.11p and IEEE 802.11ad) for radar purposes in monostatic [10]-[13] and bi-static [14] operation. Similarly, DFRC base stations (BSs) operating in sub-6 GHz and millimeterwave (mmWave) bands can concurrently transmit communication symbols to downlink users and process the backscattered signals for target detection [1], [8].

As the de facto standard for most wireless communications systems, orthogonal frequency-division multiplexing (OFDM) waveform has been widely considered for implementing DFRC systems due to its favorable characteristics, such as robustness to multipath effects, simple synchronization and highaccuracy radar operation [2], [5], [14]-[18]. While OFDM DFRC systems have certain drawbacks from a practical point of view, such as high peak-to-average power ratio (PAPR) and limitations on unambiguous range due to cyclic prefix, various techniques have been exploited to overcome these challenges, such as Golay and Reed-Muller coding [19, Ch. 3.6.3] [20] and tone reservation [21] for PAPR mitigation, and a modified pulse compression scheme to extend unambiguous range [22, Ch. 6.3.3]. To improve radar-communications tradeoff performance in OFDM DFRC systems, a multitude of waveform design ${ }^{1}$ algorithms have been proposed by jointly considering radar and communications performance metrics [18], [23]-[27]. In [18], [26], OFDM subcarrier powers are designed to simultaneously maximize the data rate and the mutual information (MI) between target impulse response and received signal. Similarly, to optimize the achievable rate while guaranteeing a certain level of radar estimation accuracy, [23] employs the Cramér-Rao bound (CRB) for target parameter estimation as the radar metric. As an alternative dual-functional approach, recent works investigate joint subcarrier selection and power allocation strategies that assign non-overlapping subcarriers to radar and communications subsystems [24], [25], [27]. The common observation in this area of research is that trade-off waveforms with optimized subcarrier powers can offer non-negligible gains over the conventional uniform power allocation strategy in terms of both target estimation performance and communications data rate.

In the prior approaches to OFDM DFRC waveform design [18], [23]-[27], including those that focus solely on the radar performance of OFDM (e.g, [28], [29]), several major limitations exist that may incur performance degradations in practical scenarios. First, these schemes typically require the knowledge of radar channel gain, which leads to online optimization of radar probing waveforms and thus brings additional computational complexity. In addition, such methods consider the design of frequency-domain waveforms without addressing the time-domain variation of subcarrier powers across consecutive symbols of an OFDM frame, which would limit Doppler estimation performance [10], [16], [19], [30]. Furthermore, characterization of waveform side-lobe levels

${ }^{1}$ In compliance with the literature, OFDM waveform design refers explicitly to power allocation across subcarriers throughout the text. 
with random communication data has been rarely studied (e.g., [15], [31]) and combined time-frequency design of an OFDM waveform with rigorous side-lobe control in the presence of random symbols has been unexplored in the literature.

Besides the above shortcomings of existing studies concerning the radar functionality of OFDM, a significant challenge involving the communications aspect of OFDM in dual-functional scenarios pertains to the availability of limited feedforward channel from the DFRC transceiver to the communications receiver [32], [33], [34], which, to the best of authors' knowledge, has not been addressed in the existing DFRC research. The key consideration is that DFRC waveforms can be designed flexibly at the transceiver side in line with the requirements of radar and communications subsystems; nevertheless, such waveforms become part of the effective channel between the transmitter and the receiver, which comprises transmit waveform (i.e., subcarrier powers) and communication channel gains. In order for the receiver to be able to decode transmit symbols, DFRC waveform information should thus be conveyed to the receiver side over a separate, possibly low-rate, feedforward/control channel [32], [34]. Therefore, DFRC waveform design algorithms should take into account the limits of feedforward channels, especially in the case of rapidly time-varying waveforms.

\section{B. Contributions}

In this paper, we propose a novel time-frequency waveform design framework for OFDM DFRC systems assuming the availability of a limited feedforward channel between the transmitter and the receiver. Leveraging tools from differenceof-convex functions (DC) programming [35] and fractional optimization [36], we introduce algorithms for designing both radar-optimal and DFRC trade-off waveforms. Unlike frequency-only waveform optimization [18], [23]-[27], we provide time-frequency waveform design algorithms to offer flexibility in the Doppler domain, which enables improved Doppler estimation. In addition, to achieve dual-functionality with high data rates, we propose the concept of limited feedforward waveform design that jointly optimizes the transmitted DFRC waveform and the feedforward/control information sent to the receiver. The main contributions can be summarized as follows:

- Radar-Optimal OFDM Waveform Design: The radaroptimal waveform design problem is formulated to optimize the delay-Doppler ambiguity function (AF) via CRB minimization for main-lobe shaping and by imposing integrated side-lobe level (ISL) constraints for side-lobe shaping, where the expected AF under random transmit symbols [15], [31] is used to quantify ISL. This leads to a challenging non-convex optimization problem with a quadratic-over-quartic fractional objective and a quadratic-fractional ISL constraint. We recast the problem using second-order cone (SOC) and difference-of-convex (DC) function constraints and develop a convex-concave procedure (CCP) based algorithm that entails solving a series of SOC programs. The proposed formulation has the advantage of being independent of target parameters and therefore allows offline optimization in a single-target scenario.

- LMMSE Based Communications Metric: To quantify the capacity loss associated with the availability of a limited feedforward channel for conveying the knowledge of time-varying subcarrier powers to the receiver, we derive a novel communications metric as a function of both the transmit waveform and the control signaling information, that provides a lower-bound on capacity by exploiting its relation to the linear minimum mean-squared-error (LMMSE) covariance matrix [37], [38]. Our approach herein is similar in spirit to that followed in multiuser multiple-input multiple-output (MIMO) broadcast scenarios for transmit beamforming [32], where effective channels are unknown at the receivers.

- OFDM DFRC Trade-off Design with Limited Feedforward: Under the assumption of a limited feedforward channel, we formulate a joint DFRC waveform and control signaling optimization (JWCSO) problem to maximize the LMMSE-based communications metric subject to a similarity constraint for the radar-optimal waveform. Capitalizing on the sparsity and rank-one structure of waveforms, respectively, at the radar and communications end of the trade-off, we propose an alternating optimization approach that iterates between updating the waveform and the control signaling information.

Additionally, simulation results indicate that the radaroptimal waveforms can outperform the traditional uniform and water-filling solutions in terms of range and velocity accuracies. Moreover, it is shown that the proposed DFRC trade-off waveforms obtained via JWCSO strategy in the presence of limited feedforward can operate very close to the boundary of the CRB-capacity region achievable with unlimited feedforward, whereas waveforms without feedforward optimization lead to significant capacity loss. The advantage of the JWCSO approach becomes more pronounced at high SNRs, where capacity improvements up to $3 \mathrm{bits} / \mathrm{s} / \mathrm{Hz}$ over feedforward-agnostic waveforms can be achieved for a given radar CRB level. Furthermore, for a given capacity level, the proposed time-varying waveforms are demonstrated to achieve lower CRB in both range and velocity than the traditional timeinvariant waveforms [18], [23]-[27], while requiring the same level of feedforward overhead. Based on the simulation results, we provide guidelines on when sparsity and rank-one structure of DFRC waveforms should be enforced depending on SNR and radar similarity regimes. We also depict the resulting waveforms in delay and Doppler domains to provide qualitative insights into the characteristics of radar and communications subsystems.

\section{SYSTEM MODEL}

Consider an OFDM DFRC transceiver equipped with a DFRC transmitter and a radar receiver operating on a single hardware platform. As shown in Fig. 1, the DFRC transceiver concurrently transmits data symbols to an OFDM communications receiver and performs radar sensing using the backscattered signals to estimate parameters of targets in the environment [5], [7]. Being co-located with the transmitter in a joint system, the radar receiver is assumed to have perfect knowledge of the transmit signal [5]. In addition, we assume perfectly decoupled transmit and receive antennas so that the radar receiver does not suffer from self-interference due to fullduplex radar operation [5], [10], [12], [16 $]^{2}$. In this section, we first provide OFDM transmit signal model and then derive signal models at the radar and communications receivers.

${ }^{2}$ There exist many self-interference cancellation techniques to achieve sufficient isolation between the transmit and receive antennas [12], [39]. For instance, in a full-duplex OFDM radar implementation, adaptive filtering based nonlinear digital cancellers in combination with RF cancellers and circulators have been shown to provide around $100 \mathrm{~dB}$ TX/RX isolation [40]. 


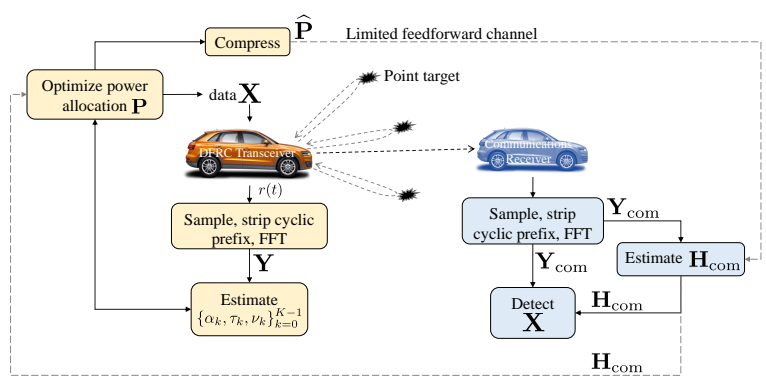

Fig. 1. Considered scenario with an OFDM DFRC transceiver and a communications receiver, where the transceiver uses the backscattered signal to detect targets. The proposed power allocation accounts for both radar and communications performance metrics.

\section{A. Transmit Signal Model}

We consider an OFDM communication signal consisting of $M$ OFDM symbols, each of which has a total duration of $T_{\mathrm{sym}}=T_{\mathrm{cp}}+T$ and a total bandwidth of $N \Delta f=B$. Here, $T_{\mathrm{cp}}$ and $T$ denote, respectively, the cyclic prefix duration and the elementary symbol duration, $\Delta f=1 / T$ is the subcarrier spacing, and $N$ is the number of subcarriers [5]. Then, the complex baseband transmit signal for the $m^{\text {th }}$ symbol is given by

$$
s_{m}(t)=\sum_{n=0}^{N-1} \sqrt{P_{n, m}} x_{n, m} e^{j 2 \pi n \Delta f t} \operatorname{rect}\left(\frac{t-m T_{\mathrm{sym}}}{T_{\mathrm{sym}}}\right),
$$

where $P_{n, m}$ and $x_{n, m}$ denote, respectively, the transmit power and the complex data symbol on the $n^{\text {th }}$ subcarrier for the $m^{\text {th }}$ symbol [17], and rect $(t)$ is a rectangular function that takes the value 1 for $t \in[0,1]$ and 0 otherwise. Denoting by $f_{c}$ the carrier frequency, the upconverted signal over the block of $M$ symbols for $t \in\left[0, M T_{\text {sym }}\right]$ can be written as

$$
\widetilde{s}(t)=\Re\left\{\sum_{m=0}^{M-1} s_{m}(t) e^{j 2 \pi f_{c} t}\right\} .
$$

\section{B. Radar Receiver}

Suppose there exist $K$ point targets in the environment, each characterized by a complex channel gain $h_{k}$ (including path loss and radar cross section), a round-trip delay $\tau_{k}$, and a normalized Doppler shift $\nu_{k} \triangleq 2 v_{k} / c$, with $v_{k}$ and $c$ denoting the radial velocity and the speed of propagation, respectively. Then, the backscattered signal at the radar receiver after downconversion can be expressed as [41], [42]

$$
r(t)=\sum_{k=0}^{K-1} \sum_{m=0}^{M-1} h_{k} s_{m}\left(t-\tau_{k}\right) e^{j 2 \pi f_{c} \nu_{k} t}+\omega(t),
$$

where $\omega(t)$ denotes the signal-independent interference plus noise term. Following [41], [42], we assume that the Dopplerinduced phase rotation within an OFDM symbol duration is negligible ${ }^{3}$, i.e., $f_{c} \nu_{k} T_{\text {sym }} \ll 1$, so that $f_{c} \nu_{k} t \approx f_{c} \nu_{k} m T_{\text {sym }}$

${ }^{3}$ This is tantamount to the requirement that the subcarrier spacing is much larger than the maximum Doppler shift, i.e., $f_{c} \nu_{k} \ll 1 / T_{\text {sym }}<1 / T=$ $\Delta f$ [16, Eq. (3.145)]. For instance, consider a DFRC base station [1] for a drone application, where $f_{c}=2.7 \mathrm{GHz}$ and $\Delta f=30 \mathrm{kHz}$, we have $f_{c} \nu_{k} T_{\mathrm{sym}}=0.0225$ for $T_{\mathrm{cp}}=T / 4$ and for a target with velocity $v_{k}=$ $30 \mathrm{~m} / \mathrm{s}$. Similarly, a mmWave vehicular application using IEEE 802.11ad [12] with $f_{c}=60 \mathrm{GHz}$ and $\Delta f=5.15625 \mathrm{MHz}$ yields $f_{c} \nu_{k} T_{\text {sym }}=0.0029$ [30] for $t \in\left[m T_{\mathrm{sym}},(m+1) T_{\mathrm{sym}}\right]$. Based on this approximation, we rewrite (3) as

$$
r(t)=\sum_{k=0}^{K-1} \sum_{m=0}^{M-1} h_{k} s_{m}\left(t-\tau_{k}\right) e^{j 2 \pi f_{c} \nu_{k} m T_{\mathrm{sym}}}+\omega(t) .
$$

Following the standard processing approach for OFDM signals [41], [42], [43, Ch. 12.1], the Fourier transform of the received signal in (4) over the elementary symbol duration (i.e., after removing the cyclic prefix) for the $m^{\text {th }}$ OFDM symbol in the $n^{\text {th }}$ subcarrier is given by

$$
y_{n, m}=\int_{m T_{\mathrm{sym}}+T_{\mathrm{cp}}}^{(m+1) T_{\mathrm{sym}}} r(t) e^{-j 2 \pi n \Delta f t} \mathrm{~d} t .
$$

To ensure orthogonality of subcarriers in (5), the cyclic prefix duration is assumed to be larger than the round-trip delay of the furthermost scatterer [2], [15], [16], i.e., $T_{\mathrm{cp}} \geq$ $\max \left\{\tau_{0}, \ldots, \tau_{K-1}\right\}$. Under this assumption, plugging (1) and (4) into (5), we have

$$
\begin{aligned}
y_{n, m}= & \sum_{k=0}^{K-1} \sum_{\bar{n}=0}^{N-1}\left(h_{k} \sqrt{P_{\bar{n}, m}} x_{\bar{n}, m} e^{-j 2 \pi \bar{n} \Delta f \tau_{k}} e^{j 2 \pi f_{c} \nu_{k} m T_{\mathrm{sym}}}\right. \\
& \left.\times \int_{m T_{\mathrm{sym}}+T_{\mathrm{cp}}}^{(m+1) T_{\mathrm{sym}}} e^{j 2 \pi(\bar{n}-n) \Delta f t} \mathrm{~d} t\right)+z_{n, m} \\
= & \sqrt{P_{n, m}} x_{n, m} \sum_{k=0}^{K-1} \alpha_{k} e^{-j 2 \pi n \Delta f \tau_{k}} e^{j 2 \pi f_{c} \nu_{k} m T_{\mathrm{sym}}} \\
& +z_{n, m}
\end{aligned}
$$

where $z_{n, m} \triangleq \int_{m T_{\mathrm{svm}}+T_{\mathrm{cp}}}^{(m+1) T_{\mathrm{sym}}} \omega(t) e^{-j 2 \pi n \Delta f t} \mathrm{~d} t$ and $\alpha_{k} \triangleq T h_{k}$. To obtain (7) from (6), we rely on the subcarrier orthogonality, i.e.,

$$
\int_{m T_{\mathrm{sym}}+T_{\mathrm{cp}}}^{(m+1) T_{\mathrm{sym}}} e^{j 2 \pi(\bar{n}-n) \Delta f t} \mathrm{~d} t=T \delta(\bar{n}-n) .
$$

For the convenience of analysis, let us define, respectively, the frequency-domain steering vector and the temporal steering vector as

$$
\begin{aligned}
& \boldsymbol{\theta}(\tau) \triangleq\left[1, e^{-j 2 \pi \Delta f \tau}, \ldots, e^{-j 2 \pi(N-1) \Delta f \tau}\right]^{T}, \\
& \boldsymbol{\phi}(\nu) \triangleq\left[1, e^{-j 2 \pi f_{c} T_{\mathrm{sym}} \nu}, \ldots, e^{-j 2 \pi f_{c}(M-1) T_{\mathrm{sym}} \nu}\right]^{T} .
\end{aligned}
$$

Accordingly, the radar observations in (7) over the block of $M$ symbols can be rewritten in a compact form as

$$
\mathbf{Y}=\underbrace{\mathbf{P} \odot \mathbf{X}}_{\substack{\text { Transmit } \\
\text { Signal }}} \odot \underbrace{\sum_{k=0}^{K-1} \alpha_{k} \boldsymbol{\theta}\left(\tau_{k}\right) \boldsymbol{\phi}^{H}\left(\nu_{k}\right)}_{\text {Radar Channel }}+\underbrace{\mathbf{Z}}_{\begin{array}{c}
\text { Noise }+ \\
\text { Interf. }
\end{array}},
$$

where $\odot$ denotes the Hadamard product, $\mathbf{Y} \in \mathbb{C}^{N \times M}, \mathbf{P} \in$ $\mathbb{R}^{N \times M}, \mathbf{X} \in \mathbb{C}^{N \times M}$ and $\mathbf{Z} \in \mathbb{C}^{N \times M}$ with $[\mathbf{Y}]_{n, m} \triangleq y_{n, m}$, $[\mathbf{P}]_{n, m} \triangleq \sqrt{P_{n, m}},[\mathbf{X}]_{n, m} \triangleq x_{n, m}$ and $[\mathbf{Z}]_{n, m} \triangleq z_{n, m}$, with $[\cdot]_{n, m}$ representing the $(n, m)^{\text {th }}$ entry of a matrix. We assume that the transmit symbols are drawn from a complex circularly-symmetric Gaussian codebook with vec $(\mathbf{X}) \sim$ $\mathcal{C N}\left(\mathbf{0}_{N M}, \mathbf{I}_{N M}\right)$, where $\operatorname{vec}(\cdot)$ denotes the matrix vectorization operator, $\mathbf{0}_{N}$ is the all-zeros vector of size $N$ and $\mathbf{I}_{N}$ is the $N \times N$ identity matrix. In addition, the interferenceplus-noise term is assumed to be distributed according to $\operatorname{vec}(\mathbf{Z}) \sim \mathcal{C N}\left(\mathbf{0}_{N M}, \mathbf{R}_{z}\right)$, where we assume the covariance matrix $\mathbf{R}_{z} \in \mathbb{C}^{N M \times N M}$ to be estimated through secondary data [44]. 
Given the transmit symbols $\mathbf{X}$, the problem of interest for radar sensing is to estimate from the observation $\mathbf{Y}$ in (11) the parameters of the radar channel, i.e., the unknown target parameters consisting of channel gains $\left\{\alpha_{k}\right\}_{k=0}^{K-1}$, delays $\left\{\tau_{k}\right\}_{k=0}^{K-1}$ and Doppler shifts $\left\{\nu_{k}\right\}_{k=0}^{K-1}$.

\section{Communications Receiver}

Following the structure in (11), the signal model at the communication side can be expressed as

$$
\mathbf{Y}_{\mathrm{com}}=\mathbf{P} \odot \mathbf{X} \odot \mathbf{H}_{\mathrm{com}}+\mathbf{Z}_{\mathrm{com}},
$$

where $\mathbf{H}_{\text {com }} \in \mathbb{C}^{N \times M}$ represents the communication channel in the time-frequency domain and $\mathbf{Z}_{\mathrm{com}}$ is the receiver noise with $\operatorname{vec}\left(\mathbf{Z}_{\text {com }}\right) \sim \mathcal{C N}\left(\mathbf{0}_{N M}, \sigma^{2} \mathbf{I}_{N M}\right)$. In accordance with the radar-communications literature [2], [10], [12], we adopt a frequency-selective communication channel model which is time-invariant during the transmission of a single block of $M$ consecutive OFDM symbols (i.e., a quasi-static block-fading channel [43, Ch. 4.3.1], [45]):

$$
\mathbf{H}_{\mathrm{com}}=\mathbf{h}_{\mathrm{com}} \mathbf{1}_{M}^{T},
$$

where $\mathbf{h}_{\text {com }} \in \mathbb{C}^{N \times 1}$ represents the channel frequency response and $\mathbf{1}_{M}$ is the all-ones vector of size $M$. The blockfading assumption in (13) can be particularly relevant for mmWave vehicular scenarios, where the transmitting and receiving vehicles are moving in the same direction, resulting in a small relative velocity and thus a negligible Doppler shift [12]. Equipped with the knowledge of $\mathbf{h}_{\text {com }}$ in (13) through pilot symbols [46] and that of $\mathbf{P}$ via control signaling [47], the communications receiver aims to decode the transmit symbols $\mathbf{X}$ from $\mathbf{Y}_{\text {com }}$ in (12).

\section{Dual-Functional Waveform Design Problem}

Our problem of interest in this paper is to design the dual-functional time-frequency waveform $\mathbf{P}$ that ensures a favorable radar-communications trade-off by joint optimization of radar and communication functionalities characterized through signal models in (11) and (12)-(13), respectively. We start by providing the performance objectives for radar and communications in Sec. III. Then, we devise algorithms for designing radar-optimal and radar-communications trade-off waveforms in Sec. IV and Sec. V, respectively.

\section{RADAR AND COMMUNICATIONS PERFORMANCE METRICS}

In this section, we derive performance metrics for OFDM radar and communication functionalities to be employed in waveform optimization. The reader is referred to the supplementary material for detailed derivations and proofs.

\section{A. Radar Performance Metric}

Assuming a noise-dominated scenario (i.e., $\mathbf{R}_{z}=\sigma_{r}^{2} \mathbf{I}_{N M}$ ) with a single-target, we consider the CRBs on delay and Doppler estimation as our estimation performance criterion [48]. According to Sec. S-I in the supplementary material, the CRBs can be obtained as

$$
\operatorname{var}(\widehat{\tau}) \geq C_{\tau}=\frac{1}{2 \gamma_{\mathrm{SNR}}} \frac{\left(\mathbf{1}^{T} \mathbf{q}\right)\left(\mathbf{q}^{T} \mathbf{\Upsilon}_{\nu} \mathbf{q}\right)}{\left(\mathbf{q}^{T} \mathbf{\Upsilon}_{\tau} \mathbf{q}\right)\left(\mathbf{q}^{T} \mathbf{\Upsilon}_{\nu} \mathbf{q}\right)-\left(\mathbf{q}^{T} \mathbf{\Upsilon}_{\tau \nu} \mathbf{q}\right)^{2}}
$$

$\operatorname{var}(\widehat{\nu}) \geq C_{\nu}=\frac{1}{2 \gamma_{\mathrm{SNR}}} \frac{\left(\mathbf{1}^{T} \mathbf{q}\right)\left(\mathbf{q}^{T} \mathbf{\Upsilon}_{\tau} \mathbf{q}\right)}{\left(\mathbf{q}^{T} \mathbf{\Upsilon}_{\tau} \mathbf{q}\right)\left(\mathbf{q}^{T} \mathbf{\Upsilon}_{\nu} \mathbf{q}\right)-\left(\mathbf{q}^{T} \mathbf{\Upsilon}_{\tau \nu} \mathbf{q}\right)^{2}}$

where

$$
\mathbf{q} \triangleq \operatorname{vec}(\mathbf{P} \odot \mathbf{P})
$$

is the equivalent optimization variable, $\widehat{\tau}$ and $\widehat{\nu}$ are unbiased estimates of $\tau$ and $\nu$, respectively, and $\gamma_{\mathrm{SNR}}, \boldsymbol{\Upsilon}_{\tau} \in$ $\mathbb{R}^{N M \times N M}, \boldsymbol{\Upsilon}_{\nu} \in \mathbb{R}^{N M \times N M}, \mathbf{\Upsilon}_{\tau \nu} \in \mathbb{R}^{N M \times N M}$ are given in (S21) in the supplementary material. As seen from (14a) and (14b), the CRBs depend on SNR, q, $\Delta f, T_{\text {sym }}$ and $f_{c}$, but they are independent of target delay-Doppler values. To design the radar-optimal waveform $\mathbf{q}$ based on a scalar metric, we consider weighted minimization of delay and Doppler CRBs in (14), which is given by

$$
f_{\mathrm{rad}}(\mathbf{q})=\lambda_{\tau} C_{\tau}+\left(1-\lambda_{\tau}\right) C_{\nu}
$$

where $\lambda_{\tau}$ and $1-\lambda_{\tau}$ denote prescribed, user-determined weighting factors for delay and Doppler estimation accuracies, with $0 \leq \lambda_{\tau} \leq 1$.

\section{B. Communications Performance Metric}

We first rewrite the received signal model in (12) in vector form as

$$
\mathbf{y}_{\mathrm{com}}=\mathbf{x} \odot \overbrace{\mathbf{p} \odot\left(\mathbf{1}_{M} \otimes \mathbf{h}_{\mathrm{com}}\right)}^{\triangleq \mathbf{h}(\mathbf{p})}+\mathbf{z}_{\mathrm{com}}=\mathbf{H}(\mathbf{p}) \mathbf{x}+\mathbf{z}_{\mathrm{com}},
$$

where $\otimes$ denotes the Kronecker product, $\mathbf{y}_{\text {com }} \triangleq$ $\operatorname{vec}\left(\mathbf{Y}_{\text {com }}\right) \in \mathbb{C}^{N M \times 1}, \mathbf{p} \triangleq \operatorname{vec}(\mathbf{P}) \in \mathbb{R}^{N M \times 1}, \mathbf{x} \triangleq$ $\operatorname{vec}(\mathbf{X}) \in \mathbb{C}^{N M \times 1}, \mathbf{z}_{\text {com }} \triangleq \operatorname{vec}\left(\mathbf{Z}_{\text {com }}\right) \in \mathbb{C}^{N M \times 1}, \mathbf{h}(\mathbf{p}) \in$ $\mathbb{C}^{N M \times 1}$ represents the effective channel experienced by the receiver, which depends on the waveform $\mathbf{p}$, and $\mathbf{H}(\mathbf{p}) \triangleq$ $\operatorname{diag}(\mathbf{h}(\mathbf{p})) \in \mathbb{C}^{N M \times N M}$, with $\operatorname{diag}(\cdot)$ returning a diagonal matrix with the elements of a vector on the diagonals.

For amplitude modulation schemes, such as pulse amplitude modulation (PAM) or quadrature amplitude modulation (QAM), estimating $\mathbf{x}$ from $\mathbf{y}_{\text {com }}$ in (17) requires the knowledge of both $\mathbf{h}_{\text {com }}$ and $\mathbf{p}$ (i.e., the effective channel gain) at the receiver. It is assumed that $\mathbf{h}_{\text {com }}$ is available at the receiver through training sequences and at the transmitter via a feedback channel [46]. As the optimization of the DFRC waveform $\mathbf{p}$ is performed at the transmitter side, this information must be conveyed to the receiver over a limited feedforward channel $^{4}$ for symbol decoding at the receiver. Following the models in [32], [34], we assume the existence of a low-rate feedforward channel (which is separate from the main communication channel) to send the control information regarding $\mathbf{p}$ to the receiver.

To characterize the capacity loss in the presence of a limited feedforward, we consider an imperfect channel state information (CSI) at the receiver, denoted by $\mathbf{H}(\widehat{\mathbf{p}})$, that results from an imperfect/partial knowledge of the transmit powers over a limited feedforward channel, denoted by $\widehat{\mathbf{p}} \in \mathbb{R}^{N M \times 1}$. Under this setting, the receiver computes the imperfectly implemented

\footnotetext{
${ }^{4}$ Unlike the conventional OFDM power allocation schemes (e.g., [49]), p varies over both frequency and time domains to fulfill the radar functionality (i.e., optimization of radar delay-Doppler characteristics) and thus may not be fully available at the receiver due to high control signaling overhead [33]. Specifically, perfect knowledge of $\mathbf{p} \in \mathbb{R}^{N M \times 1}$ at the receiver would require transmitting a control information half the amount of the user data $\mathbf{x} \in \mathbb{C}^{N M \times 1}$, which is highly impractical.
} 
[50] LMMSE estimate of $\mathbf{x}$ in (17) with $\mathbf{H}(\widehat{\mathbf{p}})$ in place of $\mathbf{H}(\mathbf{p})$ [51, p. 389]

$$
\widehat{\mathbf{x}}=\mathbf{R}_{\mathbf{x}} \mathbf{H}^{H}(\widehat{\mathbf{p}})\left(\mathbf{H}(\widehat{\mathbf{p}}) \mathbf{R}_{\mathbf{x}} \mathbf{H}^{H}(\widehat{\mathbf{p}})+\mathbf{R}_{\mathbf{z}_{\mathrm{com}}}\right)^{-1} \mathbf{y}_{\mathrm{com}},
$$

where the input and noise covariance matrices are given by $\mathbf{R}_{\mathbf{x}}=\mathbf{I}$ and $\mathbf{R}_{\mathbf{z}_{\mathrm{com}}}=\sigma^{2} \mathbf{I}$. In (18), the transmitter employs the optimal waveform $\mathbf{p}$ (see (17)), whereas the receiver performs symbol decoding using the forwarded information $\widehat{\mathbf{p}}$ [32]. It is worth highlighting that $\mathbf{p}$ is the DFRC waveform transmitted over the main channel for the purpose of joint communications and sensing, while $\widehat{\mathbf{p}}$ is sent over a separate, low-rate feedforward/control channel. Leveraging the relation between the channel capacity and the LMMSE covariance matrix [37], [38], we derive a performance metric that accounts for the availability of a limited feedforward channel, as stated in the following proposition.

Proposition 1. The capacity $C(\mathbf{p}, \widehat{\mathbf{p}})$ of the frequencyselective channel represented by the input-output relation (17) in the presence of an imperfect knowledge $\widehat{p}$ of the subcarrier powers $\mathbf{p}$ at the receiver can be lower-bounded as [37], [38], [52]

$$
C(\mathbf{p}, \widehat{\mathbf{p}}) \geq-\log \operatorname{det} \mathbf{R}_{\mathrm{LMMSE}}(\mathbf{p}, \widehat{\mathbf{p}}),
$$

where

$$
\begin{aligned}
\mathbf{R}_{\text {LMMSE }}(\mathbf{p}, \widehat{\mathbf{p}})=\operatorname{diag} & \left(\left\{\frac{\left|h_{i}(\widehat{\mathbf{p}})\right|^{2}\left(\left|h_{i}(\mathbf{p})\right|^{2}+\sigma^{2}\right)}{\left(\left|h_{i}(\widehat{\mathbf{p}})\right|^{2}+\sigma^{2}\right)^{2}}\right.\right. \\
& \left.\left.-\frac{2 \Re\left\{h_{i}^{*}(\widehat{\mathbf{p}}) h_{i}(\mathbf{p})\right\}}{\left|h_{i}(\widehat{\mathbf{p}})\right|^{2}+\sigma^{2}}+1\right\}_{i=0}^{N M-1}\right)
\end{aligned}
$$

represents the covariance matrix of the LMMSE estimate of $\mathbf{x}$ in (18), with $h_{i}(\mathbf{p}) \triangleq[\mathbf{h}(\mathbf{p})]_{i}$.

Proof. See Sec. S-II in the supplementary material.

Remark 1 (Variations in Power Allocation). We assume that the DFRC transmitter changes its power allocation $\mathbf{p}$ after each OFDM frame (consisting of $M$ symbols) to adapt to possible channel variations. In this setting, there might be gaps between practically achievable rates due to operation in the finite blocklength regime [53] and the capacity lower-bound in (19), which has been derived with an infinite blocklength assumption (as in, e.g., [54]-[57]). Despite such gaps between theoretical and practical achievable rates, adopting (19) as the optimization metric can provide useful design guidelines to improve transmission rate [43, Ch. 9.7] and offer valuable insights into radar-communications trade-off characteristics [44], [58], [59] (as will be shown in Sec. VI-B). To obtain metrics that can quantify the practical performance of such a communication system, achievable rates in the finite blocklength regime should be investigated [53], which, however, is outside the scope of this paper and can be considered for future studies.

Proposition 1 enables joint optimization of transmit waveform $\mathbf{p}$ and control signaling information $\widehat{\mathbf{p}}$, as will be discussed in Sec. V. Since the input and noise covariance matrices in (18) are diagonal, $\mathbf{R}_{\mathrm{LMMSE}}(\mathbf{p}, \widehat{\mathbf{p}})$ becomes a diagonal matrix, as well. This implies that the channel in (17) decouples into $N \times M$ single-input single-output (SISO) channels in the frequency-time blocks, allowing the capacity lower-bound in (19) to be expressed as a sum of the capacities of all individual SISO channels (see (38) in Sec. V-A). We now provide the following remarks on some special cases regarding Proposition 1.

Remark 2 (Unlimited Feedforward Channel). Given the DFRC waveform $\mathbf{p}$, the optimal control signaling strategy over an unlimited feedforward channel is to transmit the waveform itself, as expected, i.e.,

$$
\mathbf{p}=\arg \min _{\widehat{\mathbf{p}}} \log \operatorname{det} \mathbf{R}_{\mathrm{LMMSE}}(\mathbf{p}, \widehat{\mathbf{p}}) .
$$

Remark 3 (Connection to Water-filling). Under the assumption of an unlimited feedforward channel, i.e., $\widehat{\mathbf{p}}=\mathbf{p}$ (perfect CSI at the receiver), the problem of maximizing the lower bound in (19) boils down to the classical achievable rate maximization problem, i.e.,

$\min _{\mathbf{p}} \log \operatorname{det} \mathbf{R}_{\mathrm{LMMSE}}(\mathbf{p}, \mathbf{p}) \equiv \max _{\mathbf{p}} \sum_{i=0}^{N M-1} \log \left(1+\frac{\left|h_{i}(\mathbf{p})\right|^{2}}{\sigma^{2}}\right)$,

whose optimal solution under a total power constraint is given by frequency-domain water-filling with respect to the subchannel gains $\mathbf{h}_{\text {com }}$ [43, Ch. 4.4.1].

\section{RADAR-OPTIMAL OFDM WAVEFORM DESIGN WITH DELAY-DOPPLER AMBIGUITY SHAPING}

In this section, we focus on the design of radar-optimal OFDM time-frequency waveform. We first introduce an ISL constraint in the delay-Doppler ambiguity domain and formulate the waveform design problem. Then, exploiting the special problem structure, we propose a CCP based algorithm by converting the original problem into a series of SOC programs.

\section{A. Delay-Doppler Ambiguity Constraint}

Generally speaking, waveform design based on the CRB metric can produce a sharp peak in the main-lobe of the delayDoppler ambiguity domain and provide improved estimation accuracy [60]. However, the CRB characterizes only the local behavior of the delay-Doppler ambiguity function $(\mathrm{AF})$ around the origin, which might lead to high side-lobe levels if not properly accounted for in the optimization process [61]. To take into account both local (high SNR) and non-local (low SNR) estimation errors, we enforce the normalized integrated side-lobe level (ISL) constraint as [62], [63]

$$
\frac{\iint_{\mathcal{R}_{s}}|\chi(\tau, \nu)|^{2} \mathrm{~d} \tau \mathrm{d} \nu}{\left|\mathcal{R}_{s}\right||\chi(0,0)|^{2}} \leq \beta_{\mathrm{ISL}}
$$

where $\chi(\tau, \nu)$ is the $\mathrm{AF}$ of the OFDM waveform at a given delay mismatch $\tau$ and Doppler mismatch $\nu, \mathcal{R}_{s}$ denotes the side-lobe region in the delay-Doppler domain (which can be chosen flexibly according to design requirements) with the area $\left|\mathcal{R}_{s}\right|$, and $\beta_{\text {ISL }}$ is the upper limit on the allowable ISL. The constraint (22) allows us to control the relative amount of energy in 2-D portions of interest $\mathcal{R}_{s}$ of the ambiguity surface with respect to its peak, thus avoiding undesired sidelobe levels.

Recalling the observation model in (11), the AF (averaged over the distribution of transmit symbols ${ }^{5}$ ) can be derived by computing the output of the matched filter tuned to a delayDoppler pair $\left(\tau_{1}, \nu_{1}\right)$

$$
\mathbf{P} \odot \mathbf{X} \odot \boldsymbol{\theta}\left(\tau_{1}\right) \boldsymbol{\phi}^{H}\left(\nu_{1}\right),
$$

${ }^{5}$ Please refer to Sec. S-V-B in the supplementary material for details on how random data symbols $\mathbf{X}$ can impact the $\mathrm{AF}$ side-lobes. 
applied to the interference-plus-noise-free observation matrix corresponding to a target at a delay-Doppler bin $\left(\tau_{2}, \nu_{2}\right)$

$$
\mathbf{W} \odot \mathbf{P} \odot \mathbf{X} \odot \boldsymbol{\theta}\left(\tau_{2}\right) \boldsymbol{\phi}^{H}\left(\nu_{2}\right),
$$

as shown in (25) on top of page 7. In (24), $\mathbf{W} \in \mathbb{R}^{N \times M}$ represents a predefined 2-D time-frequency window ${ }^{6}$, commonly employed in radar processing to suppress side-lobes caused by rectangular windowing [64, Ch. 5.3.1]. After algebraic manipulations on (25) and defining $\mathbf{w}=\operatorname{vec}(\mathbf{W})$, we obtain

$$
\begin{aligned}
& \chi\left(\tau_{1}, \nu_{1}, \tau_{2}, \nu_{2}\right) \\
& =\mathbf{q}^{T}\left(\mathbf{w} \odot\left(\phi\left(\nu_{1}\right) \otimes \boldsymbol{\theta}^{*}\left(\tau_{1}\right)\right) \odot\left(\boldsymbol{\phi}^{*}\left(\nu_{2}\right) \otimes \boldsymbol{\theta}\left(\tau_{2}\right)\right)\right) \\
& =\mathbf{q}^{T}\left(\mathbf{w} \odot\left[\left(\phi\left(\nu_{1}\right) \odot \boldsymbol{\phi}^{*}\left(\nu_{2}\right)\right) \otimes\left(\boldsymbol{\theta}^{*}\left(\tau_{1}\right) \odot \boldsymbol{\theta}\left(\tau_{2}\right)\right)\right]\right) \\
& =\mathbf{q}^{T}\left(\mathbf{w} \odot\left(\boldsymbol{\phi}^{*}(\nu) \otimes \boldsymbol{\theta}(\tau)\right)\right) \triangleq \chi(\tau, \nu)
\end{aligned}
$$

for the delay-Doppler mismatch $(\tau, \nu) \triangleq\left(\tau_{2}-\tau_{1}, \nu_{2}-\nu_{1}\right)$.

Substituting (26) into (22), the normalized ISL constraint can be written as

$$
\begin{aligned}
& \frac{\iint_{\mathcal{R}_{s}} \mathbf{q}^{T}\left(\mathbf{w w}^{T} \odot\left(\boldsymbol{\phi}^{*}(\nu) \otimes \boldsymbol{\theta}(\tau)\right)\left(\boldsymbol{\phi}^{T}(\nu) \otimes \boldsymbol{\theta}^{H}(\tau)\right)\right) \mathbf{q} \mathrm{d} \tau \mathrm{d} \nu}{\left|\mathcal{R}_{s}\right| \mathbf{q}^{T}\left(\mathbf{w w}^{T} \odot\left(\boldsymbol{\phi}^{*}(0) \otimes \boldsymbol{\theta}(0)\right)\left(\boldsymbol{\phi}^{T}(0) \otimes \boldsymbol{\theta}^{H}(0)\right)\right) \mathbf{q}} \\
& =\frac{\mathbf{q}^{T} \boldsymbol{\Xi} \mathbf{q}}{\left|\mathcal{R}_{s}\right|\left(\mathbf{w}^{T} \mathbf{q}\right)^{2}} \leq \beta_{\mathrm{ISL}},
\end{aligned}
$$

where

$$
\Xi \triangleq \mathbf{w} \mathbf{w}^{T} \odot \iint_{\mathcal{R}_{s}} \boldsymbol{\phi}^{*}(\nu) \boldsymbol{\phi}^{T}(\nu) \otimes \boldsymbol{\theta}(\tau) \boldsymbol{\theta}^{H}(\tau) \mathrm{d} \tau \mathrm{d} \nu .
$$

\section{B. Problem Formulation}

Under the normalized ISL constraint (27) and a total power budget $\mathbf{1}^{T} \mathbf{q}=P_{T}$, the radar-optimal OFDM waveform design problem via weighted minimization of delay and Doppler CRBs in (16) can be formulated as follows:

$$
\begin{array}{cl}
\min _{\mathbf{q}} & \frac{\mathbf{q}^{T}\left[\lambda_{\tau} \boldsymbol{\Upsilon}_{\nu}+\left(1-\lambda_{\tau}\right) \mathbf{\Upsilon}_{\tau}\right] \mathbf{q}}{\left(\mathbf{q}^{T} \boldsymbol{\Upsilon}_{\tau} \mathbf{q}\right)\left(\mathbf{q}^{T} \boldsymbol{\Upsilon}_{\nu} \mathbf{q}\right)-\left(\mathbf{q}^{T} \boldsymbol{\Upsilon}_{\tau \nu} \mathbf{q}\right)^{2}} \\
\text { s.t. } & \mathbf{1}^{T} \mathbf{q}=P_{T}, \mathbf{q} \succeq \mathbf{0} \\
& \frac{\mathbf{q}^{T} \boldsymbol{\Xi} \mathbf{q}}{\left(\mathbf{w}^{T} \mathbf{q}\right)^{2}} \leq \widetilde{\beta}_{\text {ISL }},
\end{array}
$$

where we drop the immaterial scaling factor $\frac{P_{T}}{2 \gamma_{\mathrm{SNR}}}$ in (16) and define $\widetilde{\beta}_{\text {ISL }} \triangleq \beta_{\text {ISL }}\left|\mathcal{R}_{s}\right|$. In the proposed design framework (29), the level of parameter uncertainty (e.g., tracking error covariance in track radars [61]) characterizes the side-lobe region $\mathcal{R}_{s}$ in (22), while the measurement SNR governs the maximum tolerable ISL level $\beta_{\mathrm{ISL}}{ }^{7}$. For fixed $\mathcal{R}_{s}$ and $\beta_{\mathrm{ISL}}$, (29) is an offline optimization problem since the objective (29a) does not depend on target parameters, as noted in Sec. III-A.

Regarding the structure of (29), we point out the following observations: $(i)$ the objective (29a) has a highly non-convex fractional form with quadratic and quartic functions on the

${ }^{6}$ Note that $\mathbf{W}$ has no effect on the CRB since the CRB derivation is based on the time-frequency observation matrix (11) and is therefore independent of the receive processing applied afterwards. On the other hand, $\mathbf{P}$ is a dualfunctional transmit waveform that can impact both radar and communications performance, as discussed in Sec. III.

${ }^{7}$ For instance, in the case of high uncertainty regarding delay and Doppler parameters, the system designer can set $\mathcal{R}_{s}$ to cover a relatively large region in the delay-Doppler domain over which occurrence of spurious peaks can be prevented. Similarly, for low SNR operation, $\beta_{\text {ISL }}$ can be reduced to decrease the side-lobe levels so that the estimator does not choose an incorrect delayDoppler pair corresponding to a side-lobe. numerator and denominator, respectively; and, (ii) the ISL constraint $(29 \mathrm{c})$ contains a non-convex quadratic-fractional function. Hence, (29) is a challenging non-convex optimization problem that is intractable to solve to global optimality. In what follows, we provide an equivalent reformulation of (29) in terms of second-order cones (SOCs) [65] and difference-ofconvex (DC) [35] functions, that lends itself to application of efficient local optimization algorithms.

\section{Reformulation of (29) with SOC and DC Constraints}

To demonstrate the equivalent form of (29), we provide the following proposition.

Proposition 2. The waveform design problem in (29) can be equivalently reformulated as follows:

$$
\begin{aligned}
\min _{\substack{\mathbf{q}, t_{\tau}, \widetilde{t}_{\tau}, t_{\nu}, \widetilde{t}_{\nu}, \kappa_{\nu}, \kappa_{\tau \nu} \\
\text { s.t. }}} & \lambda_{\tau} t_{\tau}+\left(1-\lambda_{\tau}\right) t_{\nu} \\
& \left\|\left[\begin{array}{ll}
2 & t_{\tau}-\widetilde{t}_{\tau}
\end{array}\right]^{T}\right\|_{2} \leq t_{\tau}+\widetilde{t}_{\tau} \\
& \left\|\left[\begin{array}{ll}
2 & t_{\nu}-\widetilde{t}_{\nu}
\end{array}\right]^{T}\right\|_{2} \leq t_{\nu}+\widetilde{t}_{\nu} \\
& \left\|\left[\begin{array}{ll}
2 \kappa_{\tau \nu} & \kappa_{\nu}-\kappa_{\tau}+\widetilde{t}_{\tau}
\end{array}\right]^{T}\right\|_{2} \leq \kappa_{\nu}+\kappa_{\tau}-\widetilde{t}_{\tau} \\
& \left\|\left[\begin{array}{ll}
2 \kappa_{\tau \nu} \quad \kappa_{\tau}-\kappa_{\nu}+\widetilde{t}_{\nu}
\end{array}\right]^{T}\right\|_{2} \leq \kappa_{\tau}+\kappa_{\nu}-\widetilde{t}_{\nu} \\
& \left\|\left[\begin{array}{c}
2 \widetilde{\mathbf{u}}_{\tau}^{T} \mathbf{q} \\
1-P_{T} \mathbf{u}_{\tau}^{T} \mathbf{q}+\kappa_{\tau}
\end{array}\right]\right\|_{2} \leq 1+P_{T} \mathbf{u}_{\tau}^{T} \mathbf{q}-\kappa_{\tau} \\
& \left\|\left[\begin{array}{c}
2 \widetilde{\mathbf{u}}_{\nu}^{T} \mathbf{q} \\
1-P_{T} \mathbf{u}_{\nu}^{T} \mathbf{q}+\kappa_{\nu}
\end{array}\right]\right\|_{2} \leq 1+P_{T} \mathbf{u}_{\nu}^{T} \mathbf{q}-\kappa_{\nu} \\
& f_{1}(\mathbf{r})-f_{2}(\mathbf{r}) \leq 0 \\
& f_{3}(\mathbf{r})-f_{4}(\mathbf{r}) \leq 0 \\
& f_{4}(\mathbf{r})-f_{5}(\mathbf{r}) \leq 0,
\end{aligned}
$$

where $\mathbf{r} \triangleq\left[\mathbf{q}^{T}, \kappa_{\tau \nu}\right]^{T}$ and

$$
\begin{aligned}
& f_{1}(\mathbf{r}) \triangleq \mathbf{q}^{T} \boldsymbol{\Xi} \mathbf{q}, \quad f_{2}(\mathbf{r}) \triangleq \widetilde{\beta}_{\mathrm{ISL}}\left(\mathbf{w}^{T} \mathbf{q}\right)^{2} \\
& f_{3}(\mathbf{r}) \triangleq\left(\widetilde{\mathbf{u}}_{\tau}^{T} \mathbf{q}\right)^{2}+\left(\widetilde{\mathbf{u}}_{\nu}^{T} \mathbf{q}\right)^{2}+2\left(P_{T} \mathbf{u}_{\tau \nu}^{T} \mathbf{q}-\kappa_{\tau \nu}\right) \\
& f_{4}(\mathbf{r}) \triangleq\left(\widetilde{\mathbf{u}}_{\tau}^{T} \mathbf{q}+\widetilde{\mathbf{u}}_{\nu}^{T} \mathbf{q}\right)^{2} \\
& f_{5}(\mathbf{r}) \triangleq\left(\widetilde{\mathbf{u}}_{\tau}^{T} \mathbf{q}\right)^{2}+\left(\widetilde{\mathbf{u}}_{\nu}^{T} \mathbf{q}\right)^{2}+2\left(P_{T} \mathbf{u}_{\tau \nu}^{T} \mathbf{q}+\kappa_{\tau \nu}\right) .
\end{aligned}
$$

Proof. See Sec. S-III in the supplementary material.

As observed from Proposition 2, the equivalent problem (30) consists of a linear objective (30a), linear constraints (29b), SOC constraints (30b)-(30g), and DC type inequality constraints (30h)-(30j). Hence, the non-convexity of (30) stems from the DC functions. In the following part, we propose a convex-concave procedure (CCP) [35] based low-complexity iterative algorithm to handle this non-convexity.

\section{CCP Based Iterative Algorithm}

1) Convexification of DC Constraints: $\mathrm{CCP}$ is a local optimization heuristic that iteratively converges to a KarushKuhn-Tucker (KKT) point of the original non-convex problem (30) in an efficient manner [66, Thm. 4]. Starting from an initial feasible point ${ }^{8} \mathbf{r}_{0}$, we convexify the DC functions in

${ }^{8} \mathrm{~A}$ feasible point can be found by solving the ISL optimization problem in (S50) in Sec. S-VI of the supplementary material. 


$$
\begin{gathered}
\chi\left(\tau_{1}, \nu_{1}, \tau_{2}, \nu_{2}\right)=\mathbb{E}_{\mathbf{X}}\left\{\operatorname{tr}\left(\left(\mathbf{P} \odot \mathbf{X} \odot \boldsymbol{\theta}\left(\tau_{1}\right) \boldsymbol{\phi}^{H}\left(\nu_{1}\right)\right)^{H}\left(\mathbf{W} \odot \mathbf{P} \odot \mathbf{X} \odot \boldsymbol{\theta}\left(\tau_{2}\right) \boldsymbol{\phi}^{H}\left(\nu_{2}\right)\right)\right)\right\} \\
=\operatorname{tr}\left((\mathbf{P} \odot \mathbf{P})^{T}(\underbrace{\mathbb{E}_{\mathbf{X}}\left\{\mathbf{X}^{*} \odot \mathbf{X}\right\}}_{\mathbf{1}_{N} \mathbf{1}_{M}^{T}} \odot \mathbf{W} \odot \boldsymbol{\theta}^{*}\left(\tau_{1}\right) \boldsymbol{\phi}^{T}\left(\nu_{1}\right) \odot \boldsymbol{\theta}\left(\tau_{2}\right) \boldsymbol{\phi}^{H}\left(\nu_{2}\right))\right)=\mathbf{q}^{T} \operatorname{vec}\left(\mathbf{W} \odot \boldsymbol{\theta}^{*}\left(\tau_{1}\right) \boldsymbol{\phi}^{T}\left(\nu_{1}\right) \odot \boldsymbol{\theta}\left(\tau_{2}\right) \boldsymbol{\phi}^{H}\left(\nu_{2}\right)\right) .
\end{gathered}
$$

(30h)-(30j) by linearizing the concave part around the current iterate $\mathbf{r}_{\ell}=\left[\mathbf{q}_{\ell}^{T},\left(\kappa_{\tau \nu}\right)_{\ell}\right]^{T}$ as [35]

$$
f_{i}(\mathbf{r})-\widehat{f}_{j}\left(\mathbf{r} ; \mathbf{r}_{\ell}\right) \leq 0
$$

for $(i, j) \in\{(1,2),(3,4),(4,5)\}$, where $\widehat{f}_{j}\left(\mathbf{r} ; \mathbf{r}_{\ell}\right) \triangleq f_{j}\left(\mathbf{r}_{\ell}\right)+$ $\nabla f_{j}\left(\mathbf{r}_{\ell}\right)^{T}\left(\mathbf{r}-\mathbf{r}_{\ell}\right)$.

2) SOC Representation of the Convexified ISL Constraint through Vandermonde Decomposition: Defining $\mathbf{a}_{\ell} \triangleq$ $2 \widetilde{\beta}_{\mathrm{ISL}} \mathbf{w}^{T} \mathbf{q}_{\ell} \mathbf{w}$ and $\widetilde{a}_{\ell} \triangleq \widetilde{\beta}_{\mathrm{ISL}}\left(\mathbf{w}^{T} \mathbf{q}_{\ell}\right)^{2}$, the convexified ISL constraint in (32) for $(i, j)=(1,2)$ can be expressed as

$$
\mathbf{q}^{T} \boldsymbol{\Xi} \mathbf{q}-\mathbf{a}_{\ell}^{T} \mathbf{q}+\widetilde{a}_{\ell} \leq 0 .
$$

Assume that the 2-D smoothing window in (28) is given by $\mathbf{W}=\mathbf{w}_{\tau} \mathbf{w}_{\nu}^{T}$ and the side-lobe region $\mathcal{R}_{s}$ is rectangular, i.e., $\mathcal{R}_{s}=\mathcal{R}_{\tau} \times \mathcal{R}_{\nu}$, where $\mathcal{R}_{\tau}$ and $\mathcal{R}_{\nu}$ represent the corresponding delay and Doppler intervals, respectively. Let

$$
\begin{aligned}
& \boldsymbol{\Xi}_{\tau} \triangleq \int_{\mathcal{R}_{\tau}} \boldsymbol{\theta}(\tau) \boldsymbol{\theta}^{H}(\tau) \mathrm{d} \tau=\mathbf{V}_{\tau} \mathbf{D}_{\tau} \mathbf{V}_{\tau}^{H} \\
& \boldsymbol{\Xi}_{\nu} \triangleq \int_{\mathcal{R}_{\nu}} \boldsymbol{\phi}^{*}(\nu) \phi^{T}(\nu) \mathrm{d} \nu=\mathbf{V}_{\nu} \mathbf{D}_{\nu} \mathbf{V}_{\nu}^{H}
\end{aligned}
$$

denote the Vandermonde decompositions [67] of the Hermitian positive semi-definite Toeplitz matrices $\boldsymbol{\Xi}_{\tau}$ and $\boldsymbol{\Xi}_{\nu}$, respectively. Then, the ISL constraint in (33) can be reformulated as the following SOC (see Sec. S-IV in the supplementary material for details) [65, Ch. 2.1]:

$$
\left\|\left[\begin{array}{c}
2\left(\mathbf{D}_{\nu}^{1 / 2} \otimes \mathbf{D}_{\tau}^{1 / 2}\right)\left(\widetilde{\mathbf{V}}_{\nu} \otimes \widetilde{\mathbf{V}}_{\tau}\right)^{H} \mathbf{q} \\
1-\mathbf{a}_{\ell}^{T} \mathbf{q}+\widetilde{a}_{\ell}
\end{array}\right]\right\|_{2} \leq 1+\mathbf{a}_{\ell}^{T} \mathbf{q}-\widetilde{a}_{\ell},
$$

where $\widetilde{\mathbf{V}}_{\tau}=\mathbf{w}_{\tau} \mathbf{1}_{R_{\tau}}^{T} \odot \mathbf{V}_{\tau}$ and $\widetilde{\mathbf{V}}_{\nu}=\mathbf{w}_{\nu} \mathbf{1}_{R_{\nu}}^{T} \odot \mathbf{V}_{\nu}$.

3) SOCP Formulation: Reformulating all the constraints in (32) as SOCs [65, Ch. 2.1], the problem to be solved at the $\ell^{\text {th }}$ iteration of CCP becomes

$$
\begin{aligned}
& \min _{\widetilde{\tau}_{t}, \widetilde{T}_{\tau}} \lambda_{\tau} t_{\tau}+\left(1-\lambda_{\tau}\right) t_{\nu} \\
& \underset{\kappa_{\tau}, \kappa_{\nu}, \kappa_{\tau \nu}}{\mathbf{q}, t_{\tau}, \widetilde{t}_{\tau}, t_{\nu}, \widetilde{t}_{\nu}} \\
& \text { s.t. }(29 b),(30 b)-(30 g),(35) \\
& \left\|\left[2 \mathbf{q}^{T}\left[\widetilde{\mathbf{u}}_{\tau} \widetilde{\mathbf{u}}_{\nu}\right], \quad 1+\mathbf{b}_{\ell}^{T} \mathbf{q}-2 \kappa_{\tau \nu}+\widetilde{b}_{\ell}\right]^{T}\right\|_{2} \\
& \leq 1-\mathbf{b}_{\ell}^{T} \mathbf{q}+2 \kappa_{\tau \nu}-\widetilde{b}_{\ell} \\
& \left\|\left[2 \mathbf{q}^{T}\left(\widetilde{\mathbf{u}}_{\tau}+\widetilde{\mathbf{u}}_{\nu}\right), \quad 1-\mathbf{c}_{\ell}^{T} \mathbf{q}-2 \kappa_{\tau \nu}+\widetilde{c}_{\ell}\right]^{T}\right\|_{2} \\
& \leq 1+\mathbf{c}_{\ell}^{T} \mathbf{q}+2 \kappa_{\tau \nu}-\widetilde{c}_{\ell},
\end{aligned}
$$

where $\mathbf{b}_{\ell} \triangleq 2 P_{T} \mathbf{u}_{\tau \nu}-2\left(\widetilde{\mathbf{u}}_{\tau}+\widetilde{\mathbf{u}}_{\nu}\right)^{T} \mathbf{q}_{\ell}\left(\widetilde{\mathbf{u}}_{\tau}+\widetilde{\mathbf{u}}_{\nu}\right), \widetilde{b}_{\ell} \triangleq$ $\left[\left(\widetilde{\mathbf{u}}_{\tau}+\widetilde{\mathbf{u}}_{\nu}\right)^{T} \mathbf{q}_{\ell}\right]^{2}, \mathbf{c}_{\ell} \triangleq 2\left(\widetilde{\mathbf{u}}_{\tau}^{T} \mathbf{q}_{\ell} \widetilde{\mathbf{u}}_{\tau}+\widetilde{\mathbf{u}}_{\nu}^{T} \mathbf{q}_{\ell} \widetilde{\mathbf{u}}_{\nu}+P_{T} \mathbf{u}_{\tau \nu}\right)$, and $\widetilde{c}_{\ell} \triangleq\left(\widetilde{\mathbf{u}}_{\tau}^{T} \mathbf{q}_{\ell}\right)^{2}+\left(\widetilde{\mathbf{u}}_{\nu}^{T} \mathbf{q}_{\ell}\right)^{2}$.

The problem (36) is a second-order cone program (SOCP) and therefore can be solved efficiently using available conic solvers [68]. The proposed iterative algorithm for solving the problem (29) (or, equivalently, (30)) is summarized in Algorithm 1.

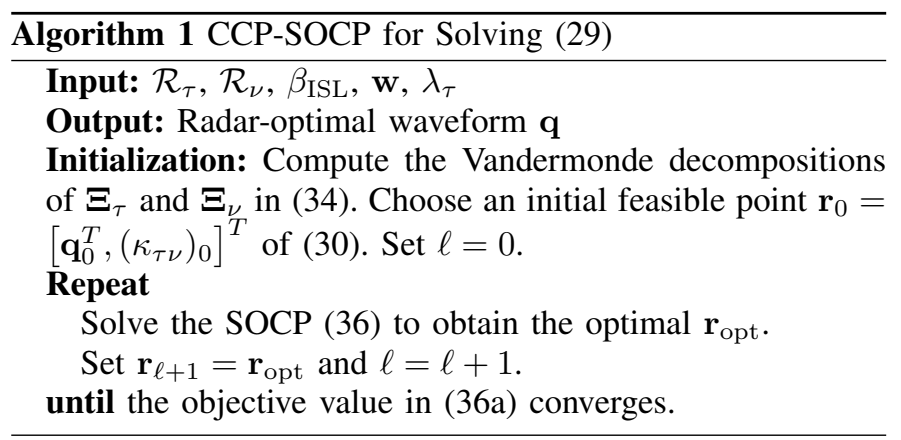

\section{RADAR-COMMUnications TRADE-OFF DESIGN With LIMITED FEEDFORWARD}

In this section, we consider radar-communications trade-off designs with limited feedforward by employing the communications objective in (19) under a constraint on the similarity to the radar-optimal waveforms designed in Sec. IV.

\section{A. Joint Waveform-Control Signaling Optimization (JWCSO)}

Given the radar-optimal waveform $\mathbf{p}_{\text {rad }}$, we formulate the trade-off waveform design problem as ${ }^{9}$

$$
\begin{array}{cl}
\min _{\mathbf{p}, \widehat{\mathbf{p}}} & \log \operatorname{det} \mathbf{R}_{\mathrm{LMMSE}}(\mathbf{p}, \widehat{\mathbf{p}}) \\
\text { s.t. } & \|\mathbf{p}\|_{2}^{2} \leq P_{T} \\
& \left\|\mathbf{p}-\mathbf{p}_{\mathrm{rad}}\right\|_{2}^{2} /\left(2 P_{T}\right) \leq \varepsilon \\
& {[\text { Control Overhead Constraint on } \widehat{\mathbf{p}}],}
\end{array}
$$

where $0 \leq \varepsilon \leq 1$ determines the level of similarity between the radar-optimal and the radar-communications tradeoff waveforms ${ }^{10}$. In (37), we perform joint optimization of the waveform $\mathbf{p}$ and the feedforward/control signaling information $\widehat{\mathbf{p}}$ to maximize the capacity lower-bound in (19) subject to a tolerable mismatch between $\mathrm{p}$ and $\mathbf{p}_{\mathrm{rad}}$, and a control signaling overhead constraint on $\widehat{\mathbf{p}}$ [33].

Let $\overline{\mathbf{h}} \triangleq \mathbf{1}_{M} \otimes \mathbf{h}_{\text {com }}$ and $\bar{h}_{i} \triangleq[\overline{\mathbf{h}}]_{i}$. Then, plugging (20) and the definition of $\mathbf{h}(\mathbf{p})$ in (17) into (37), the problem becomes

$$
\begin{array}{cl}
\max _{\mathbf{p}, \widehat{\mathbf{p}}} & \sum_{i=0}^{N M-1} \log \left(\frac{A_{i}(\mathbf{p}, \widehat{\mathbf{p}})}{B_{i}(\mathbf{p}, \widehat{\mathbf{p}})}\right) \\
\text { s.t. } & (37 \mathrm{~b})-(37 \mathrm{~d}),
\end{array}
$$

where

$$
\begin{aligned}
& A_{i}(\mathbf{p}, \widehat{\mathbf{p}}) \triangleq\left(\widehat{p}_{i}\left|\bar{h}_{i}\right|^{2}+\sigma^{2}\right)^{2} \\
& B_{i}(\mathbf{p}, \widehat{\mathbf{p}}) \triangleq\left(\widehat{p}_{i}\left|\bar{h}_{i}\right|^{2}+\sigma^{2}\right)^{2}+\widehat{p}_{i}\left|\bar{h}_{i}\right|^{2}\left(p_{i}\left|\bar{h}_{i}\right|^{2}+\sigma^{2}\right)
\end{aligned}
$$

${ }^{9}$ Since the radar-optimal waveform can be designed offline, we pursue a sequential optimization approach that designs the DFRC trade-off waveform using a similarity constraint, which provides computational savings compared to a joint strategy that optimizes radar and communications metrics in a single problem.

${ }^{10} \varepsilon$ can be chosen depending on practical requirements and priorities of radar and communications subsystems. In a radar-critical operation with stringent accuracy requirements, $\varepsilon$ can be close to 0 , while for scenarios requiring high-rate data transmission, $\varepsilon$ can be set close to 1 . 


$$
-2 \sqrt{\widehat{p}_{i} p_{i}}\left|\bar{h}_{i}\right|^{2}\left(\widehat{p}_{i}\left|\bar{h}_{i}\right|^{2}+\sigma^{2}\right) .
$$

The problem (38) is a non-convex optimization problem with a non-concave objective function in the joint variable $(\mathbf{p}, \widehat{\mathbf{p}})$. Exploiting the framework in [36], we devise an alternating optimization procedure to obtain near-optimal solutions to (38), as described in Sec. V-C.

\section{B. Limited Feedforward via Sparsity and Rank-One Con- straints}

According to Remark 2 in Sec. III-B, the optimal forwarded information $\widehat{\mathbf{p}}$ that maximizes the capacity lower-bound in (38) is the waveform $\mathbf{p}$ itself in the absence of control overhead constraints in (37d). However, such an unconstrained choice of $\widehat{p}$ incurs a large overhead on the feedforward channel as $p$ contains subcarrier powers of an entire OFDM frame. Based on this observation, we propose two strategies to enforce a special structure on $\widehat{\mathbf{p}}$ through (37d) to enable its transmission over a low-rate feedforward channel. To this end, we exploit the following observations regarding the structure of $\mathbf{p}$ :

- Sparsity Constraint: As $\varepsilon \rightarrow 0$ in (37c) (i.e., convergence to radar-optimal waveform), the optimal $\mathbf{p}$ becomes sparse in time-frequency ${ }^{11}$ (only a small percentage of subcarriers has a non-negligible power level), thereby leading to the constraint

$$
\|\widehat{\mathbf{p}}\|_{0} /(N M) \leq \zeta,
$$

where $0 \leq \zeta \leq 1$ denotes the normalized limit on the feedforward channel. In this scheme, the transmitter sends over the feedforward link the non-zero power levels and their indices ${ }^{12}$.

- Rank-One Constraint: As $\varepsilon \rightarrow 1$ in (37c) (i.e., convergence to communication-optimal waveform), the optimal $\mathbf{P}$ exhibits the rank-one structure $\mathbf{P}=\mathbf{p}_{\text {freq }} \mathbf{1}_{M}^{T}$ (equivalently, $\mathbf{p}=\mathbf{1}_{M} \otimes \mathbf{p}_{\text {freq }}$ ), where $\mathbf{p}_{\text {freq }} \in \mathbb{R}^{N \times 1}$ represents the frequency-domain waveform. As indicated in Remark 3 and as will be verified in Sec. VI-B, $\mathbf{p}_{\text {free }}$ coincides with the water-filling solution at $\varepsilon=1$. Hence, we propose to enforce the constraint

$$
\widehat{\mathbf{p}}=\mathbf{1}_{M} \otimes \mathbf{p}_{\text {freq }},
$$

where the forwarded information is $\mathbf{p}_{\text {freq }}$. In this case, the overhead ratio is given by $\zeta=1 / M$.

For limited feedforward design, the sparsity constraint in (41) or the rank-one constraint in (42) can be enforced depending on the operation regime in terms of SNR and radar similarity $\varepsilon$, which will be further elaborated on in Sec. VI-B. As a complementary mechanism to the proposed constraints, various other criteria, such as discretization, compression via run-length encoding [33], [69] and constrained entropy [69], can be applied on the optimized feedforward values obtained via (37) to further reduce the number of bits transmitted over the feedforward channel.

\footnotetext{
${ }^{11}$ This will be verified through simulations in Sec. VI. In particular, please see Fig. 2 and Fig. 9(a).

${ }^{12}$ We highlight that the LMMSE decoding in (18) and the associated capacity expression in (19) or (38) are valid for any $\widehat{\mathbf{p}}$. For instance, if some elements in $\widehat{\mathbf{p}}$ are zero due to sparsity constraint, the corresponding time-frequency bins do not contribute to the overall capacity in (38) since $\log \left(\frac{A_{i}(\mathbf{p}, \widehat{\mathbf{p}})}{B_{i}(\mathbf{p}, \widehat{\mathbf{p}})}\right)=0$ when $\widehat{p}_{i}=0$.
}

\section{Reformulation of (38) via Quadratic Transform}

The problem (38) belongs to a special class of non-convex fractional programming problems, called the sum-of-functionsof-ratio problem [36]. When $\widehat{\mathbf{p}}$ is fixed, (38) becomes a concave-convex fractional program in $\mathbf{p}$, which, although being a difficult non-convex problem, can be solved as a sequence of convex optimization problems. This motivates the use of the quadratic transform [36, Cor. 2], a generalized version of Dinkelbach's transform, to recast (38) into an equivalent form, as shown in the following lemma.

Lemma 1. The radar-communications waveform design problem in (38) can be equivalently recast as

$$
\begin{aligned}
& \max _{\mathbf{p}, \widehat{\mathbf{p}}, \boldsymbol{\varrho}} \sum_{i=0}^{N M-1} \log \left(2 \varrho_{i} \sqrt{A_{i}(\mathbf{p}, \widehat{\mathbf{p}})}-\varrho_{i}^{2} B_{i}(\mathbf{p}, \widehat{\mathbf{p}})\right) \\
& \text { s.t. }(37 \mathrm{~b}),(37 \mathrm{c}) \\
& \begin{cases}(41), & \text { if sparsity constraint on } \widehat{\mathbf{p}} \\
(42), & \text { if rank - one constraint on } \widehat{\mathbf{p}}\end{cases} \\
& \varrho_{i} \in \mathbb{R}, \quad i=0, \ldots, N M-1,
\end{aligned}
$$

with a newly introduced auxiliary variable $\varrho$. $\left.\rho_{i}^{2} B_{i}\right)$.

Proof. See [36, Cor. 2], using $A_{i} / B_{i}=\max _{\rho_{i}}\left(2 \rho_{i} \sqrt{A_{i}}-\right.$

The equivalent problem (43) is now amenable to alternating optimization that alternates over $\mathbf{p}, \widehat{\mathbf{p}}$ and $\varrho$ while holding the remaining terms fixed.

\section{Alternating Optimization for Solving (43)}

The proposed design approach is described below and summarized in Algorithm 2. Since the constraint functions of $\mathbf{p}$ and $\widehat{\mathbf{p}}$ are decoupled, Algorithm 2 converges to a KKT point of (38) under the condition that the solutions to the three subproblems are unique [70, Prop. 1].

1) Optimize $\mathbf{p}$ for Fixed $\widehat{\mathbf{p}}$ and $\varrho$ : The optimization of the trade-off waveform $\mathbf{p}$ for a given control signaling information $\widehat{\mathbf{p}}$ and auxiliary variable $\varrho$ yields the subproblem

$$
\begin{aligned}
\mathbf{p}^{\star}=\arg \max _{\mathbf{p}} & \sum_{i=0}^{N M-1} \log \left(2 \varrho_{i} \sqrt{A_{i}(\mathbf{p}, \widehat{\mathbf{p}})}-\varrho_{i}^{2} B_{i}(\mathbf{p}, \widehat{\mathbf{p}})\right) \\
\text { s.t. } & (37 \mathrm{~b}),(37 \mathrm{c}),
\end{aligned}
$$

which is a concave maximization problem [71, Ch. 3.2.4] under convex quadratic constraints and thus can be efficiently solved through convex optimization.

2) Optimize $\widehat{\mathbf{p}}$ for Fixed $\mathbf{p}$ and $\varrho$ : For the sparsity constraint in (41), the optimal $\widehat{\mathbf{p}}$ can be obtained via separability of the objective in (43) and the entries of $\widehat{\mathbf{p}}$ being decoupled as (cf. Remark 2)

$$
\widehat{p}_{i}^{\star}=\left\{\begin{array}{ll}
p_{i}, & i \in \mathcal{I} \\
0, & \text { otherwise }
\end{array},\right.
$$

where $\mathcal{I} \subseteq\{0, \ldots, N M-1\}$, with $|\mathcal{I}|=\zeta N M$, contains the indices of the subcarriers with the highest $\zeta N M$ values from $\varrho_{i}\left(p_{i}\left|\bar{h}_{i}\right|^{2}+\sigma^{2}\right)\left(2-\varrho_{i} \sigma^{2}\right) \cdot{ }^{13}$

\footnotetext{
${ }^{13}$ This can be derived by plugging $\widehat{p}_{i}=p_{i}$ into the argument of the log function in (43).
} 
For the rank-one constraint in (42), the optimal $\mathbf{p}_{\text {freq }}$ is given by

$$
\begin{aligned}
\mathbf{p}_{\text {freq }}^{\star}=\arg \max _{\mathbf{p}_{\text {freq }}} & \sum_{n=0}^{N-1} \sum_{m=0}^{M-1} \log \left(f_{n, m}\left(\mathbf{p}, p_{\text {freq }, n}, \boldsymbol{\varrho}\right)\right) \\
\text { s.t. } & \mathbf{p}_{\text {freq }} \preceq P_{T} \mathbf{1},
\end{aligned}
$$

where $\sqrt{p_{\text {freq }, n}} \triangleq\left[\mathbf{p}_{\text {freq }}\right]_{n}$,

$$
f_{n, m}\left(\mathbf{p}, p_{\text {freq }, n}, \varrho\right) \triangleq 2 \varrho_{n, m} \sqrt{A_{n, m}(\mathbf{p}, \widehat{\mathbf{p}})}-\varrho_{n, m}^{2} B_{n, m}(\mathbf{p}, \widehat{\mathbf{p}})
$$

with $\widehat{\mathbf{p}}=\mathbf{1}_{M} \otimes \mathbf{p}_{\text {freq }}$ and the vectorized index $i$ being replaced by the row-column pair $(n, m)$. The optimization problem in (46) can be decomposed into $N$ independent subproblems

$$
p_{\text {freq, }, n}^{\star}=\arg \max _{0 \leq p_{\text {freq }, n} \leq P_{T}} \sum_{m=0}^{M-1} \log \left(f_{n, m}\left(\mathbf{p}, p_{\text {freq }, n}, \varrho\right)\right)
$$

for $n=0, \ldots, N-1$, which can be solved by executing a simple line search method (e.g., Golden-section search with multiple initial points [72]). We note that (46) provides a general design strategy for $\mathbf{p}_{\text {freq }}$ which coincides with the water-filling solution at $\varepsilon=1$, but yields different solutions for $\varepsilon<1$.

3) Optimize $\varrho$ for Fixed $\mathbf{p}$ and $\widehat{\mathbf{p}}$ : For a given $\mathbf{p}$ and $\widehat{\mathbf{p}}$, the closed-form optimal solution for $\varrho$ is given by [36]

$$
\varrho_{i}^{\star}=\frac{\sqrt{A_{i}(\mathbf{p}, \widehat{\mathbf{p}})}}{B_{i}(\mathbf{p}, \widehat{\mathbf{p}})}, i=0, \ldots, N M-1 .
$$

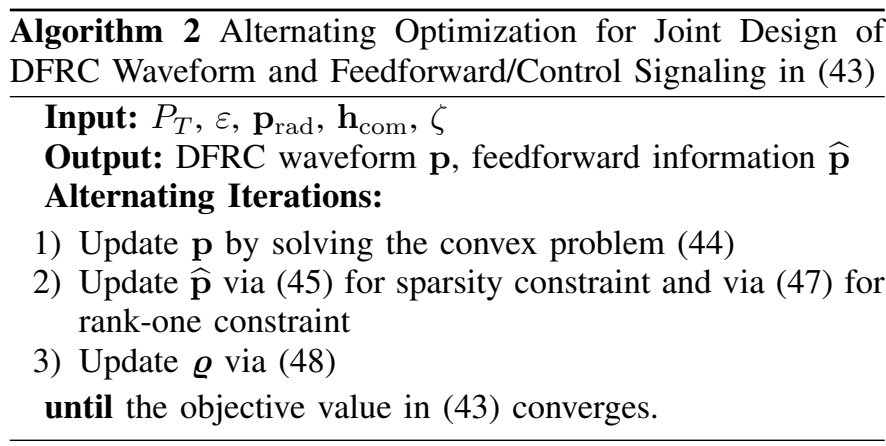

\section{E. Special Case of Unlimited Feedforward Channel}

In this part, we investigate the special case of the DFRC trade-off design problem in (37) for unlimited feedforward channels, in which case $\widehat{\mathbf{p}}=\mathbf{p}$ is the optimal feedforward information for a given waveform $\mathbf{p}$ (see Remark 2). According to Remark 3, the DFRC waveform design problem with $\widehat{\mathbf{p}}=\mathbf{p}$ reverts to

$$
\begin{array}{ll}
\max _{\mathbf{p}} & \sum_{i=0}^{N M-1} \log \left(1+\frac{p_{i}\left|\bar{h}_{i}\right|^{2}}{\sigma^{2}}\right) \\
\text { s.t. } & (37 \mathrm{~b}),(37 \mathrm{c}),
\end{array}
$$

which can be solved via convex optimization tools [68]. The problem in (49) can be highly relevant for PSK modulations since the receiver does not require the knowledge of $\mathbf{p}$ to decode transmit symbols $\mathbf{x}$ in (17) (meaning that $\mathbf{p}$ is perfectly available at the receiver). Note that the objective in (49) is mostly valid at low SNRs as a communications metric for PSK constellations and the validity region can be enlarged by increasing modulation order [73].
TABLE I

OFDM PARAMETER SETS FOR SIMULATIONS

\begin{tabular}{|l|l|l|}
\hline Parameter & Set 1 & Set 2 \\
\hline Carrier Frequency, $f_{c}$ & $2 \mathrm{GHz}$ & $60 \mathrm{GHz}$ \\
\hline Total Bandwidth, $B$ & $1.5 \mathrm{MHz}$ & $128 \mathrm{MHz}$ \\
\hline Number of Subcarriers, $N$ & 64 & 256 \\
\hline Subcarrier Spacing, $\Delta f$ & $23.4 \mathrm{kHz}$ & $500 \mathrm{kHz}$ \\
\hline Total Symbol Duration, $T_{\text {sym }}$ & $64 \mu \mathrm{s}$ & $3 \mu \mathrm{s}$ \\
\hline Number of Symbols, $M$ & 16 & 256 \\
\hline Block Duration, $M T_{\text {sym }}$ & $1.02 \mathrm{~ms}$ & $768 \mu \mathrm{s}$ \\
\hline
\end{tabular}

TABLE II

Main-Lobe Width vs. ISL Constraint For the SCEnARIo IN FIg. 3

\begin{tabular}{|l|l|l|l|l|l|l|l|}
\hline$\beta_{\text {ISL }}(\mathrm{dB})$ & -18 & -15 & -12 & -9 & -6 & -3 & 0 \\
\hline Range $(\mathrm{m})$ & 0.62 & 0.57 & 0.52 & 0.48 & 0.45 & 0.40 & 0.39 \\
\hline Velocity $(\mathrm{m} / \mathrm{s})$ & 1.71 & 1.61 & 1.49 & 1.37 & 1.23 & 1.09 & 1.09 \\
\hline
\end{tabular}

\section{Simulation Results}

In this section, we provide simulation results to illustrate the performance of the proposed waveform design algorithms. In order to exemplify both sub-6 GHz DFRC base station scenarios (e.g., 5G NR cellular systems [1]) and vehicular DFRC systems in the mmWave band [12], the two sets of OFDM parameters are used, as shown in Table I. For simulations, per-sample $\mathrm{SNR}$ is given by $\mathrm{SNR}=P_{\mathrm{avg}} / \sigma^{2}$, where $P_{\text {avg }}=P_{T} /(N M)$ is the average power constraint. The communication channel $\mathbf{h}_{\text {com }}$ in (13) is generated by using 4 and 340 taps in the delay domain, respectively, for Set 1 and Set 2, where each tap follows a zero-mean complex Gaussian distribution with variance $\sigma_{\text {com }}^{2}$ [8], [46]. In addition, we set the weighting factor in (16) as $\lambda_{\tau}=0.5$ and use the SI units $\mathrm{m}$ and $\mathrm{m} / \mathrm{s}$ for $C_{\tau}$ and $C_{\nu}$, respectively, to balance the delay and Doppler parts. Furthermore, we define the uniform waveform as the one with equal subcarrier powers, i.e., $P_{n, m}=P_{\mathrm{avg}}$, $\forall n, m$.

\section{A. Radar-Optimal Waveforms: Performance of Algorithm 1}

In this part, we present design examples from the proposed radar waveform design algorithm in Algorithm 1 and investigate its convergence behavior and delay-Doppler estimation performance by fixing the radar channel gain to $|\alpha|=0 \mathrm{~dB}$.

1) Waveform Design Examples: To illustrate the effect of the ISL constraint on the delay-Doppler ambiguity characteristics, Fig. 2 plots the optimal time-frequency waveforms obtained by solving (29) with and without the ISL constraint (29c) and the corresponding AFs. In addition, Fig. 3 demonstrates the range and velocity profiles of the optimal and uniform waveforms along with the controlled side-lobe regions. For the scenario in Fig. 3, Table II shows the mainlobe widths in range and velocity (corresponding to $-6 \mathrm{~dB} \mathrm{AF}$ magnitude) for various ISL constraints $\beta_{\mathrm{ISL}}$. It can be observed that the proposed approach with the introduced ISL constraint offers a good compromise between estimation accuracy (mainlobe sharpness) and side-lobe suppression, leading to globally optimal waveforms over $\mathcal{R}_{s}$. Moreover, the unconstrained waveform puts all the available power on the edges of the timefrequency region because the CRB is inversely proportional to RMS bandwidth and RMS envelope [74], [75]. However, such a power allocation creates aliasing (grating lobes) in the delay-Doppler spectrum due to insufficient sampling of the time-frequency region. The ISL-constrained waveform has a more balanced time-frequency distribution and can prevent high side-lobe levels in the delay-Doppler domain. 


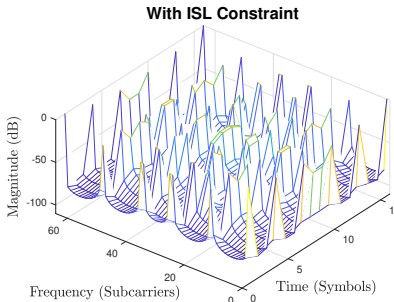

(a)

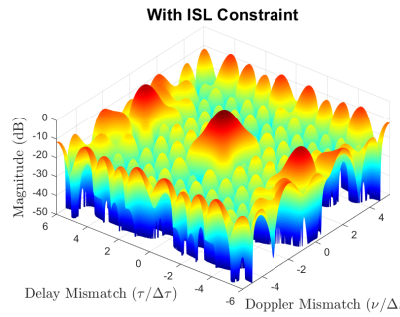

(c)

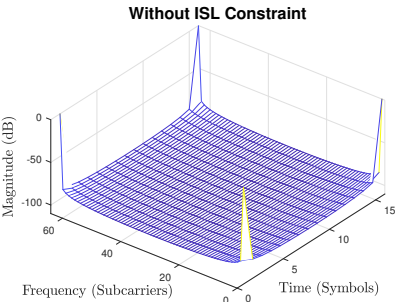

(b)

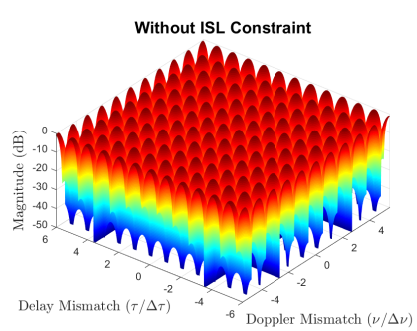

(d)
Fig. 2. Optimal time-frequency waveforms $\mathbf{q}$ obtained by solving (29) (a) with and (b) without the ISL constraint (29c), and the corresponding delayDoppler AFs in (c) and (d) computed via (26), where the OFDM parameters are shown in Table I (Set 1), $\mathbf{W}$ is the 2-D Hamming window, $\lambda_{\tau}=0.5$ $\beta_{\mathrm{ISL}}=-25 \mathrm{~dB}$, and $\mathcal{R}_{s}$ is given by $[-4,4]$ in normalized delay and Doppler mismatch values (in multiples of delay resolution $\Delta \tau$ and Doppler resolution $\Delta \nu$, respectively), excluding the main-lobe region $[-1,1]$. Ambiguity-aware design in (a) can produce a waveform with low side-lobe levels in (c) over the given side-lobe region of interest, whereas blindly optimizing CRB without side-lobe control in (b) creates ambiguities over the entire delay-Doppler domain in (d).

2) Convergence Behavior of CCP Algorithm: To showcase the convergence behavior of Algorithm 1 initialized at the uniform waveform, Fig. 4 demonstrates the evolution of the range and velocity CRBs in the objective (29a) and the ISL in $(29 \mathrm{c})$ over the CCP iterations. It is observed that Algorithm 1 converges in few iterations, with a monotonically decreasing sequence of CRB values ${ }^{14}$. Hence, by iteratively solving the SOCP in (36), the proposed CCP approach can enhance the range and velocity accuracy of radar well beyond that of the conventional uniform waveform. Moreover, we notice that the ISL converges to its upper bound while the CRBs are improving, which agrees with the fundamental trade-off between main-lobe smearing and side-lobe leakage [76, Ch. 2.6.1]. This is also corroborated by Fig. 5, which shows the evolution of waveforms with respect to the CCP iterations.

3) Impact of ISL Constraint on RMSE Performance: To evaluate the actual estimator performance, we run a Monte Carlo simulation with 1000 trials to compute the root meansquared errors (RMSEs) achieved by the maximum-likelihood estimators (MLEs) of delay and Doppler parameters:

$$
(\widehat{\tau}, \widehat{\nu}, \widehat{\alpha})=\arg \min _{\tau, \nu, \alpha}\left\|\mathbf{Y} \odot \mathbf{W}-\mathbf{P} \odot \mathbf{X} \odot \alpha \boldsymbol{\theta}(\tau) \phi^{H}(\nu)\right\|_{F}^{2},
$$

where

$$
\widehat{\alpha}=\frac{\operatorname{tr}\left(\left(\mathbf{P} \odot \mathbf{X} \odot \boldsymbol{\theta}(\tau) \boldsymbol{\phi}^{H}(\nu)\right)^{H}(\mathbf{Y} \odot \mathbf{W})\right)}{\left\|\mathbf{P} \odot \mathbf{X} \odot \boldsymbol{\theta}(\tau) \boldsymbol{\phi}^{H}(\nu)\right\|_{F}^{2}},
$$

\footnotetext{
${ }^{14}$ Each CCP iteration takes around 600 seconds to complete in MATLAB. It is worth emphasizing that the radar-optimal waveforms can be designed offline via (29), as pointed out in Sec. IV-B, which bypasses real-time processing requirements. As a low-complexity, suboptimal alternative to joint time-frequency design, we have performed separate 1-D designs for time and frequency domains using Algorithm 1 and observed similar AF characteristics, in which case CCP iterations take around 0.6 seconds.
}

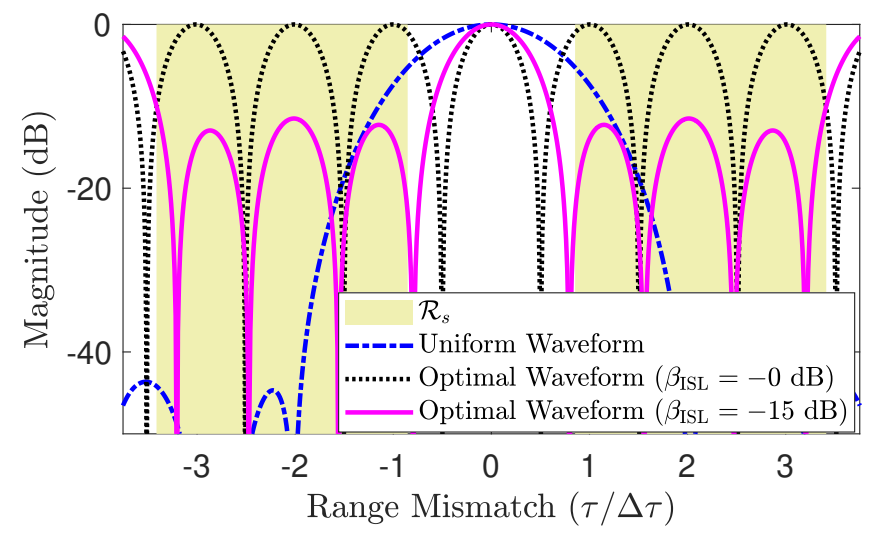

(a)

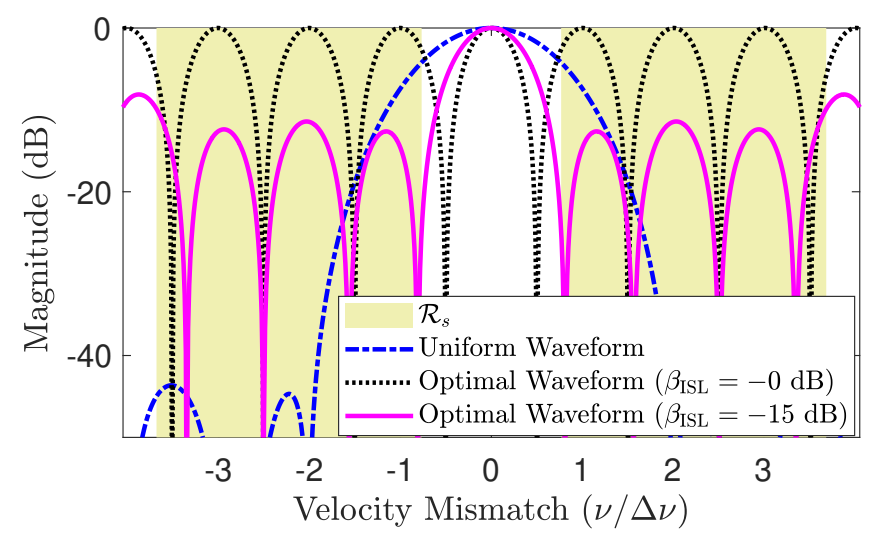

(b)

Fig. 3. (a) Range and (b) velocity profiles (i.e., zero-Doppler and zero-delay cuts of the AF in (26)) corresponding to the uniform waveform (with equal subcarrier powers) and the optimal waveforms obtained via (29) with and without the ISL constraint $(29 \mathrm{c})$ (i.e., $\beta_{\mathrm{ISL}}=0 \mathrm{~dB}$ ), where the parameters are shown in Table I (Set 2 ), $\mathcal{R}_{s}$ covers 4 resolution cells in delay and Doppler domains excluding the mainlobe region, range and velocity mismatch values are shown in terms of multiples of delay resolution $\Delta \tau$ and Doppler resolution $\Delta \nu$, respectively, and $\mathbf{W}$ is the 2-D Hamming window. The proposed waveform design strategy with a rigorous side-lobe control can sharpen the main-lobe peak with respect to the uniform waveform, leading to an improved estimation accuracy, without producing ambiguities.

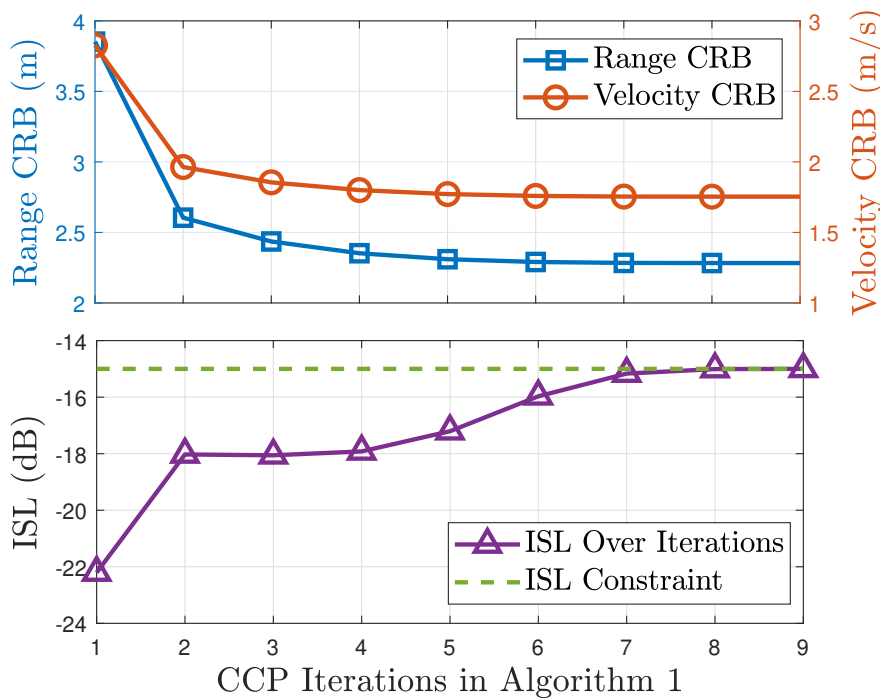

Fig. 4. Convergence behavior of Algorithm 1 for $\mathrm{SNR}=-10 \mathrm{~dB}$, initialized at the uniform waveform, using the parameters in Table I (Set 1). 


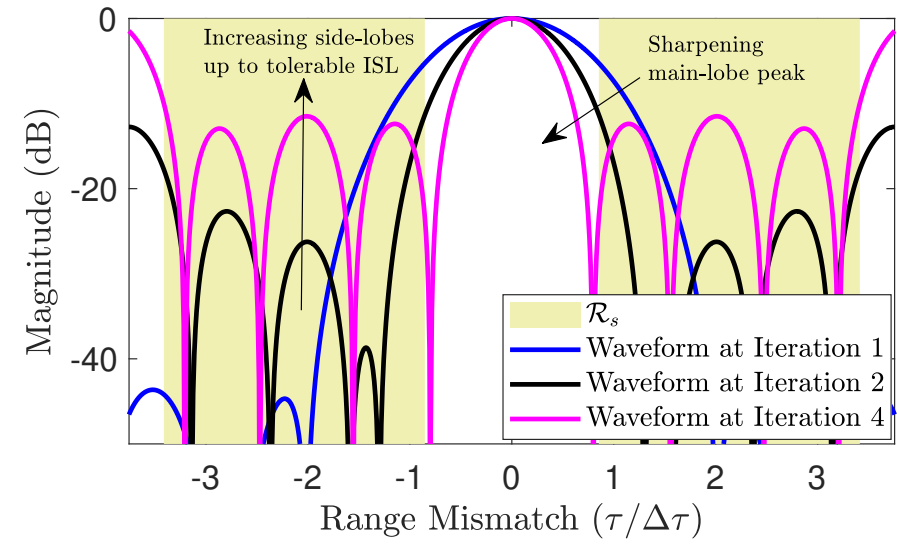

Fig. 5. Evolution of range profiles (zero-Doppler cut of the AF in (26)) of the waveforms over the CCP iterations in Algorithm 1, where the parameters are shown in Table I (Set 2) and range mismatch values are shown in terms of multiples of delay resolution $\Delta \tau$. The algorithm converges to a waveform with reduced main-lobe width (i.e., smaller CRB) and side-lobe levels raised up to the allowable ISL.
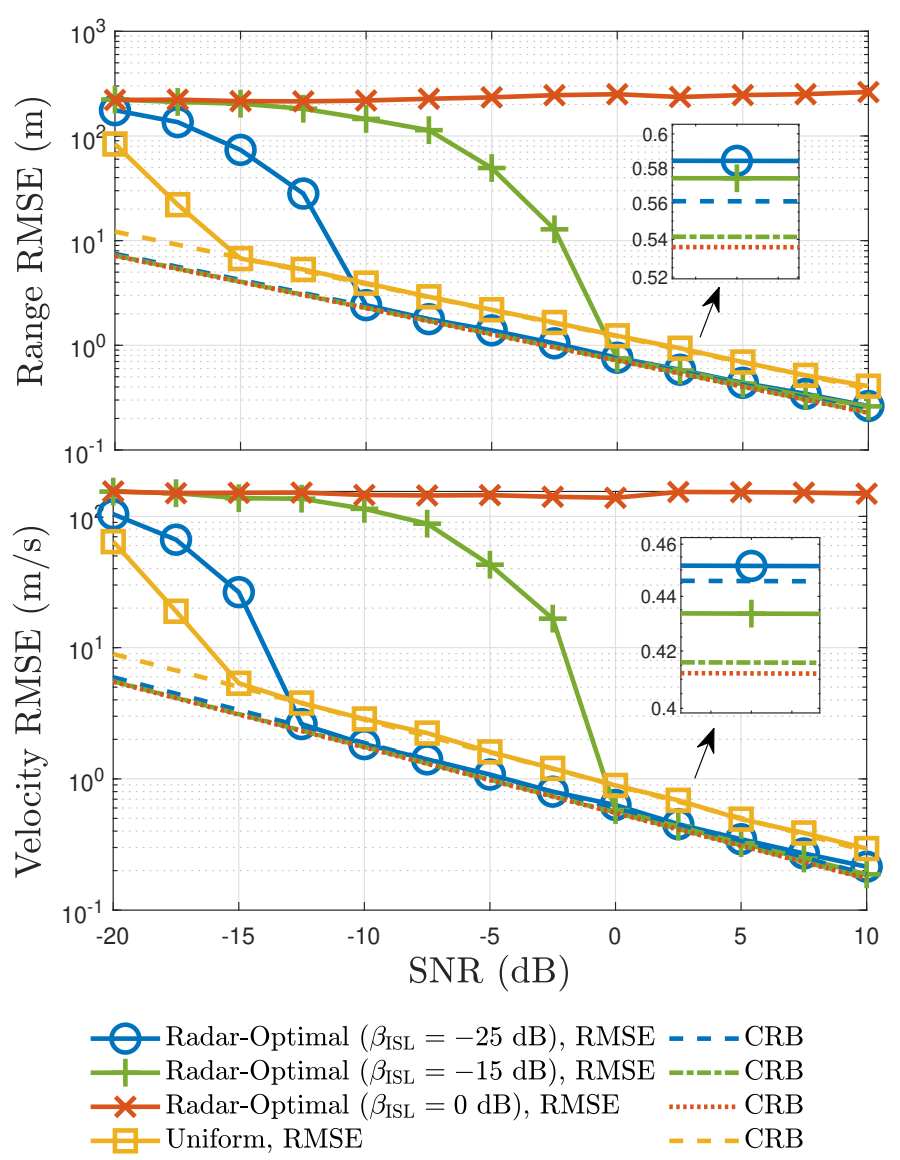

Fig. 6. Range and velocity estimation performances of the OFDM waveforms designed using Algorithm 1 with the parameters in Table I (Set 1).

and the search space for $(\tau, \nu)$ is chosen as $\mathcal{R}_{s}$ including the main-lobe region ${ }^{15}$. We compare the RMSE performance of three different strategies: (i) the uniform waveform, (ii) the radar-optimal waveform with the ISL constraint in $(29 \mathrm{c})$ $\left(\beta_{\mathrm{ISL}}=-15 \mathrm{~dB}\right.$ and $\left.\beta_{\mathrm{ISL}}=-25 \mathrm{~dB}\right)$, and (iii) the radaroptimal waveform without $(29 \mathrm{c})$ (i.e., $\beta_{\mathrm{ISL}}=0 \mathrm{~dB}$ ).

Fig. 6 plots the range and velocity RMSEs with respect to SNR, along with the corresponding CRBs. It is seen that the MLEs for the uniform waveform and the ISL-constrained

${ }^{15}$ Please refer to Sec. S-V-A in the supplementary material for details on the implementation of the MLE in (50).

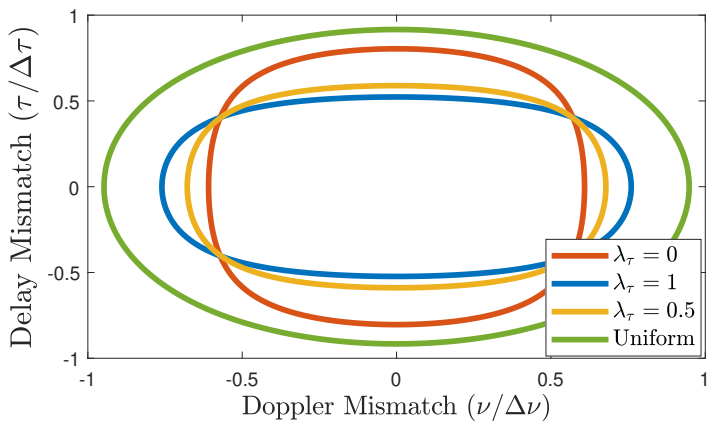

Fig. 7. $-6 \mathrm{~dB}$ contour plots of the delay-Doppler AFs corresponding to the uniform waveform and the radar-optimal waveforms obtained by solving (29) using different weighting factors $\lambda_{\tau}$ with the parameters in Table I (Set 1), where $\Delta \tau$ and $\Delta \nu$ denote, respectively, the delay and Doppler resolutions.

radar-optimal waveforms can attain the CRBs as the SNR increases, whereas the RMSE of the unconstrained radaroptimal waveform does not improve with SNR due to delayDoppler ambiguities ${ }^{16}$, in compliance with Fig. 2 and Fig. 3. This proves the significance of the ISL constraint, introduced in Sec. IV-A, in circumventing the ambiguity problem for CRBbased waveform optimization. Moreover, the ISL-constrained waveforms outperform the uniform strategy in terms of the RMSE performances above a certain SNR threshold that increases with $\beta_{\text {ISL }}$. These results reveal that the proposed approach becomes more robust to noise as the ISL constraint gets tight and is superior to the conventional uniform solution in the medium-to-high SNR regime ${ }^{17}$, which complies with tracking scenarios [61]. Futhermore, as $\beta_{\text {ISL }}$ increases, the corresponding ISL-constrained waveform achieves higher accuracy, implying that increasingly larger ISL values can be imposed as the SNR gets higher. We underline the fact that the proposed waveforms are highly flexible and can be reconfigured for very low SNR scenarios by enlarging $\mathcal{R}_{s}$ and decreasing $\beta_{\mathrm{ISL}}$ in $(29 \mathrm{c})$.

4) Trade-Off Between Delay and Doppler CRBs: We analyze the trade-off between delay and Doppler CRBs resulting from the scalarization in the radar objective (16) through the weighting factor $\lambda_{\tau}$. Fig. 7 shows the $-6 \mathrm{~dB}$ contour plots of the delay-Doppler AFs corresponding to the uniform waveform and the radar-optimal waveforms designed via (29) using different $\lambda_{\tau}$. It is observed that $\lambda_{\tau}$ governs the tradeoff between the main-lobe widths along the delay and Doppler axes. In particular, as $\lambda_{\tau}$ increases, the objective in (16) places more emphasis on the minimization of delay CRB than that of Doppler CRB, which in turn renders the peak of the AF sharper along the delay domain and more spread along the Doppler domain. Moreover, the proposed radar-optimal waveforms are superior to the uniform waveform in terms of both delay and Doppler accuracies irrespective of $\lambda_{\tau}$.

\section{B. DFRC Trade-Off Waveforms: Performance of Algorithm 2}

In this part, we illustrate the radar-communications trade-off performance of the proposed time-frequency DFRC waveform design approach in Algorithm 2 using Set 1 in Table I with $|\alpha|=0 \mathrm{~dB}$. Based on the results in Fig. 6, $\mathbf{p}_{\text {rad }}$ in $(37 \mathrm{c})$ is

${ }^{16}$ Even in the absence of noise (infinite SNR), the unconstrained waveform cannot achieve the CRB because the estimator would choose one of the secondary peaks in $\mathcal{R}_{s}$ equally likely.

${ }^{17}$ At medium SNR, the RMSE performance gap between the radar-optimal waveforms and the uniform one is on the order of $\mathrm{m}$ and $\mathrm{m} / \mathrm{s}$ in range and velocity, respectively, which can be crucial in certain applications, such as vehicular positioning [77]. 
chosen as the radar-optimal waveform designed under the ISL constraint $\beta_{\mathrm{ISL}}=-25 \mathrm{~dB}$. Since there exists no previous work on DFRC waveform design with feedforward constraints, we consider the following schemes for benchmarking purposes:

- Unlimited Feedforward Channel (UFC): Solve (49) (i.e., perfect CSI at the receiver). This scheme establishes an upper bound on the capacity for a given similarity level $\varepsilon$ in $(37 \mathrm{c})$ and incurs an overhead of $\zeta=1$.

- Joint Optimization of $\mathbf{p}$ and $\widehat{\mathbf{p}}$ with the Sparsity Constraint (JWCSO-Sparse): The proposed algorithm in Algorithm 2 using the sparsity constraint in (41) over a limited feedforward channel with an overhead $0<\zeta<1$.

- Joint Optimization of $\mathbf{p}$ and $\widehat{\mathbf{p}}$ with the Rank-One Constraint (JWCSO-RankOne): The proposed algorithm in Algorithm 2 using the rank-one constraint in (42) over a limited feedforward channel with an overhead $\zeta=1 / M$.

- Feedforward-Agnostic Optimization (FAO): The traditional waveform design approach that optimizes $\mathbf{p}$ as in the UFC scheme without feedforward optimization and uses the uniform subcarrier powers for decoding at the receiver, i.e., $\widehat{p}_{i}=P_{\text {avg }}$.

- Water-filling: Water-filling solution in Remark 3 with an overhead $\zeta=1 / M$

- UFC Time-Invariant Waveform (UFC-TI): Special case of the UFC scheme where the same frequency-domain DFRC waveform is employed over the entire OFDM frame, leading to an overhead $\zeta=1 / M$. Here, the timeinvariant radar-optimal waveform is designed by solving (29) with an additional rank-one constraint of the form $\mathbf{q}=\mathbf{1}_{M} \otimes \mathbf{q}_{N}$, where $\mathbf{q}_{N} \in \mathbb{R}^{N \times 1}$ represents the frequency-domain waveform.

In Fig. 8, we depict the achievable CRB-capacity tradeoff region along with the trade-off curves corresponding to different schemes for $\mathrm{SNR}=-10 \mathrm{~dB}$ and $\sigma_{\text {com }}^{2}=35 \mathrm{~dB}$ as the radar similarity constraint $\varepsilon$ in (37c) varies over the interval $[0,1]$. In addition, to investigate the performances of different schemes with respect to SNR, Fig. 9 plots the capacity for various radar similarity levels.

1) Capacity-CRB Trade-off Curves: From Fig. 8, it is seen that the UFC scheme delineates the boundary of the achievable trade-off region between radar estimation accuracy and communication rate, in compliance with Remark 2 in Sec. III-B. Hence, it yields the highest capacity that can be attained for a given radar similarity constraint $\varepsilon$. In the communicationoptimal regime (i.e., $\varepsilon=1$ ), the UFC coincides with the waterfilling solution, as expected.

Inspecting the FAO and UFC curves in Fig. 8 and Fig. 9, we observe that optimizing only the DFRC waveform without feedforward optimization can significantly degrade the communication performance, especially at high communication SNRs. Therefore, the traditional feedforward-agnostic (waveform-only) design approach for OFDM dual-functional systems fails to provide high data rates with time-varying subcarrier powers (i.e., when the Doppler ambiguity domain needs to be shaped). On the other hand, through joint waveform and feedforward optimization, the proposed JWCSO approach in Algorithm 2 can achieve near-optimal trade-off performance in the sense that it can almost reach the capacity upper bound of the UFC benchmark for a given similarity level $\varepsilon$ (either using JWCSO-Sparse for low $\varepsilon$ or using JWCSO-RankOne for high $\varepsilon$ ). This indicates that

- the LMMSE-based metric in Proposition 1 provides an accurate quantification of limited feedforward effects,
- the JWCSO strategy, which jointly designs $\mathbf{p}$ and $\widehat{\mathbf{p}}$, can allow the DFRC system equipped with a low-rate feedforward channel $(\zeta \ll 1)$ to operate very close to its theoretical limits attainable with unlimited feedforward $(\zeta=1)$, and

- Algorithm 2 can provide globally optimal solutions to the non-convex problem in (38).

Additionally, in the high SNR regime (Fig. 8), JWCSORankOne achieves significant capacity gain via feedforward optimization with respect to the FAO benchmark ${ }^{18}$ (up to more than 3 bits/s/Hz improvement). Comparing Fig. 8 and Fig. 9, we remark that the benefit of the proposed JWCSO approach is more prominent at high SNRs.

The trade-off curves in Fig. 8 suggest that the proposed DFRC waveforms offer a flexible trade-off between radar and communications, outperforming the conventional uniform and water-filling solutions in terms of accuracy and/or capacity. In addition, different from such conventional approaches, the proposed waveforms can be dynamically adapted to varying practical system requirements (i.e., whether it is a radar-critical scenario with $\varepsilon \approx 0$ or a communications-critical scenario with $\varepsilon \approx 1$ ). Moreover, it is seen that the range and velocity CRBs can be improved substantially with only a negligible loss in communications performance. Similarly, the DFRC waveforms can increase capacity up to a certain similarity $\varepsilon$ without degrading the range-velocity accuracy of radar.

2) Capacity With Respect to SNR: From Fig. 9, it can be observed that the JWCSO approach with the rank-one constraint consistently outperforms the FAO at all SNRs and similarity levels, closing the substantial capacity gap with respect to the UFC benchmark. In addition, the performance gain via feedforward optimization with the rank-one constraint becomes more evident as the SNR increases and the radar waveform similarity decreases (i.e., $\varepsilon$ increases).

Comparing the two variants of the JWCSO, we notice that the sparsity constraint outperforms the rank-one constraint for small $\varepsilon$, while the latter exhibits better performance as $\varepsilon \rightarrow 1$ and finally meets the water-filling solution at $\varepsilon=1$ (as seen from Fig. 8), in agreement with the observations in Sec. V-B. Moreover, the lower the SNR, the smaller the performance gap of the sparsity-based approach with respect to the UFC benchmark since the contribution of the unselected subcarriers $i \notin \mathcal{I}$ in (45) to the equivalent capacity objective in (43) is negligible at low SNRs. Nevertheless, in the high SNR regime, JWCSO-Sparse suffers from the so-called diminishing marginal returns property of the $\log$ function [81, Ch. 5.2.2]. This effect can also be noticed from Fig. 8, where the capacity achieved by JWCSO-Sparse under a high communication SNR saturates at a certain $\varepsilon$ since the unselected subcarriers provide non-negligible contribution to the overall capacity in this regime.

This observation offers valuable insights into the characteristics of radar and communication functionalities. While the radar-optimal waveform exhibits a sparse/peaky nature irrespective of the SNR, the water-filling solution converges to the uniform power allocation as the SNR increases [78][80], as seen from Fig. 8. Hence, the sparsity assumption holds at the radar side at all SNRs; however, it breaks down at the communication side in the high SNR regime. Based on the

\footnotetext{
${ }^{18}$ Note that FAO meets the water-filling solution at $\varepsilon=1$ for the high SNR scenario in Fig. 8. The reason is that FAO assumes uniform power allocation for decoding at the receiver and the optimal waveform designed by FAO at $\varepsilon=1$ coincides with the water-filling solution, which converges to the uniform power allocation at high SNRs [78]-[80].
} 


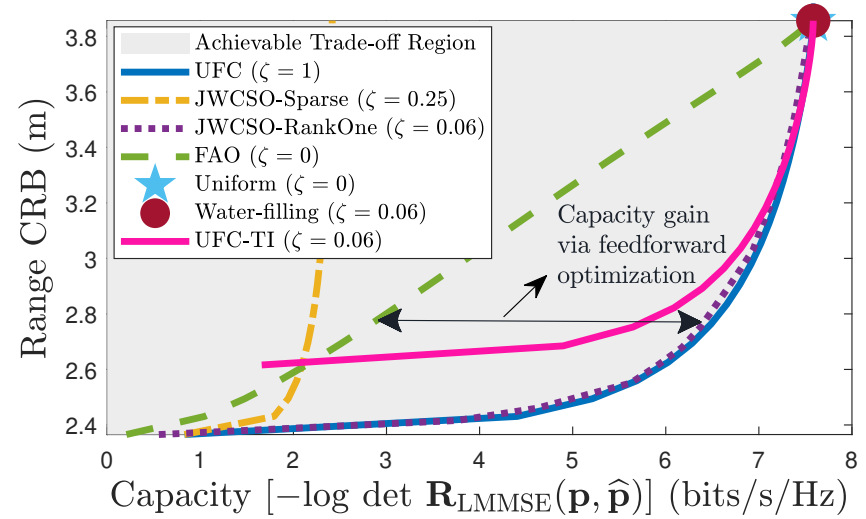

(a)

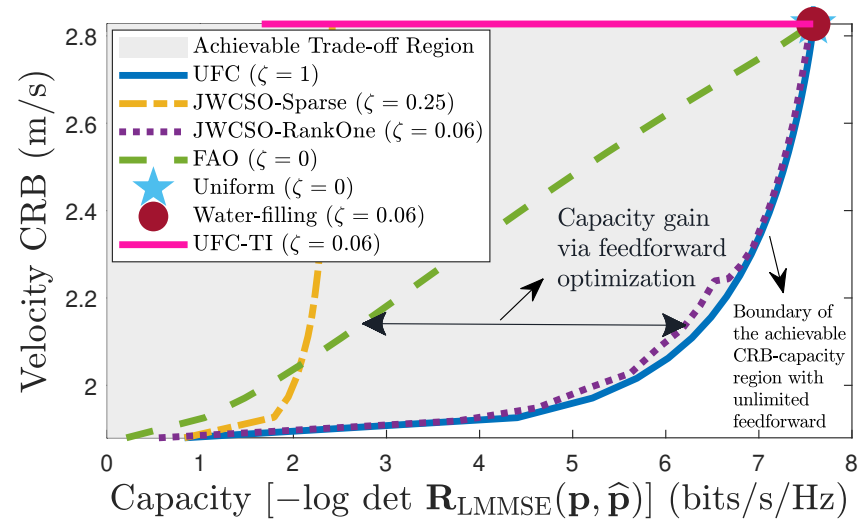

(b)

Fig. 8. Radar-communications trade-off curves obtained by different approaches as $\varepsilon$ in $(37 \mathrm{c})$ varies over $[0,1]$, where the ISL constraint of the radaroptimal waveform is $\beta_{\mathrm{ISL}}=-25 \mathrm{~dB}, \mathrm{SNR}=-10 \mathrm{~dB}, \sigma_{\text {com }}^{2}=35 \mathrm{~dB}$ and $0 \leq \zeta \leq 1$ indicates the feedforward/control overhead of the corresponding approach.

TABLE III

GUIDELINES ON Which CONSTRAINT TO ENFORCE In ALGORITHM 2 UNDER VARIOUS OPERATION REGIMES

\begin{tabular}{|l|l|l|}
\hline & Low SNR & High SNR \\
\hline Radar-Optimal $(\varepsilon \rightarrow 0)$ & Sparse & Sparse \\
\hline Weighted $(0<\varepsilon<1)$ & Sparse & Rank-One \\
\hline Communication-Optimal $(\varepsilon \rightarrow 1)$ & Rank-One & Rank-One \\
\hline
\end{tabular}

above outcomes, we provide, in Table III, a rough guideline on which variant of the JWCSO algorithm to choose at different operation regimes.

3) Time-Varying vs. Time-Invariant Design: In this part, we compare the trade-off performances of the proposed timevarying DFRC waveforms (i.e., time-frequency waveforms as discussed above) and the traditional time-invariant DFRC waveforms (e.g., [18], [23]-[27]), represented by UFC and UFC-TI schemes, respectively. From Fig. 8, it can be seen that for a given capacity level, the time-varying waveform always achieves lower CRB (i.e., higher accuracy) in both range and velocity than the time-invariant one. In addition, the velocity CRB of the time-invariant waveform cannot even be improved with respect to the uniform and water-filling solutions (due to uniform power allocation in time), while the time-varying waveform exhibits trade-off behavior in both range and velocity domains. Hence, the proposed time-varying design is superior to its time-invariant counterpart in terms of radarcommunications trade-off performance. We emphasize that both designs require the same level of feedforward overhead, i.e., $\widehat{\mathbf{p}}$ is of size $N$ (the number of subcarriers), when the JWCSO-RankOne strategy is employed for the time-varying waveform.

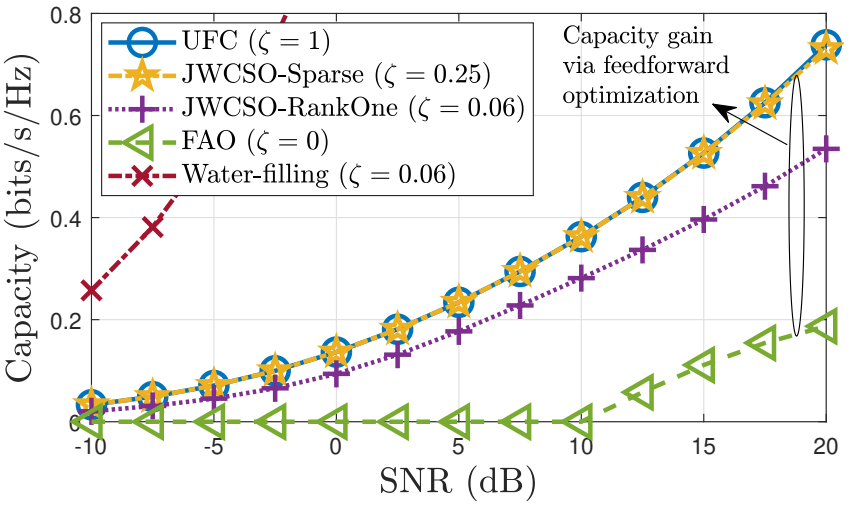

(a)

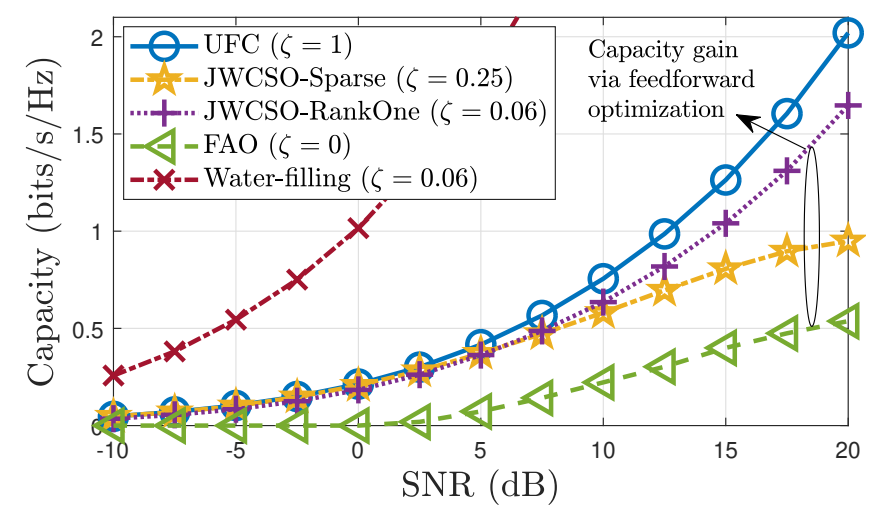

(b)

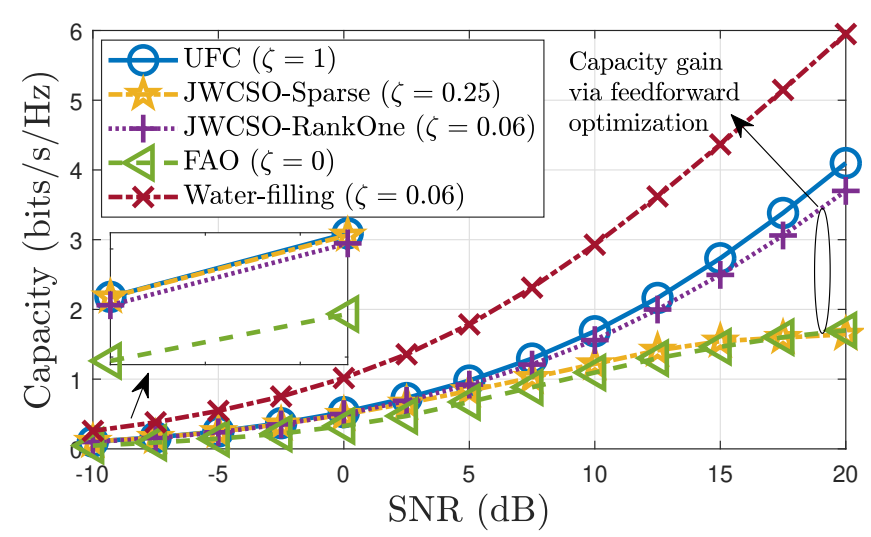

(c)

Fig. 9. Communication performance of different schemes with respect to SNR for various radar similarity levels, where $\sigma_{\text {com }}^{2}=0 \mathrm{~dB}$. (a) $\varepsilon=0.0001$. (b) $\varepsilon=0.01$. (c) $\varepsilon=0.1$.

\section{Radar-Communications Trade-off at mmWave}

In this part, we employ the parameters from Set 2 in Table I to show the trade-off between the radar estimation performance and the communications rate for a mmWave DFRC system employing the UFC scheme. In (37c), $\mathbf{p}_{\mathrm{rad}}$ is set to be the radar-optimal waveform designed under the ISL constraint $\beta_{\mathrm{ISL}}=-15 \mathrm{~dB}$. Fig. 10 illustrates the rangevelocity CRB vs. capacity curves with varying SNR levels. It is seen that cm-level accuracy can be achieved even with very weak target returns $(|\alpha|=-35 \mathrm{~dB})$, satisfying the requirements of mmWave vehicular DFRC systems [11]. In addition, the trade-off becomes more favorable with increasing SNR, as expected, which implies that the achievable rate can be increased significantly without sacrificing the radar functionality. In Fig. 11, we plot the range and velocity profiles of the OFDM waveforms with varying similarity constraints 


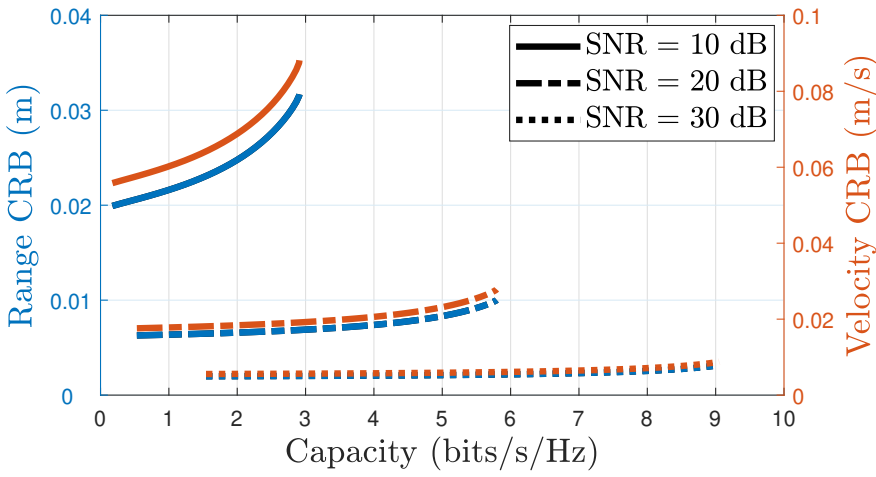

Fig. 10. Range-velocity CRB vs. capacity trade-off curves of a mmWave DFRC system at various SNR levels, where the radar channel gain is set to $|\alpha|=-35 \mathrm{~dB}$ and the ISL constraint of the radar-optimal waveform is $\beta_{\mathrm{ISL}}=-15 \mathrm{~dB}$.

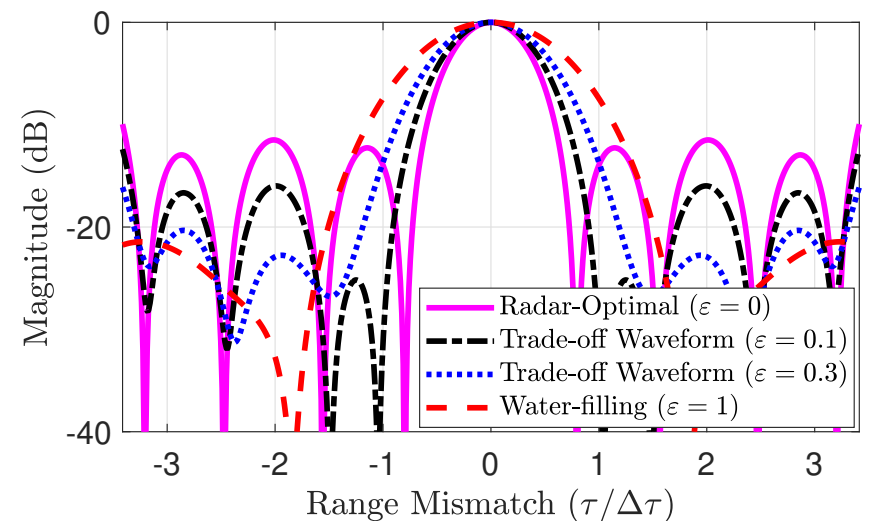

(a)

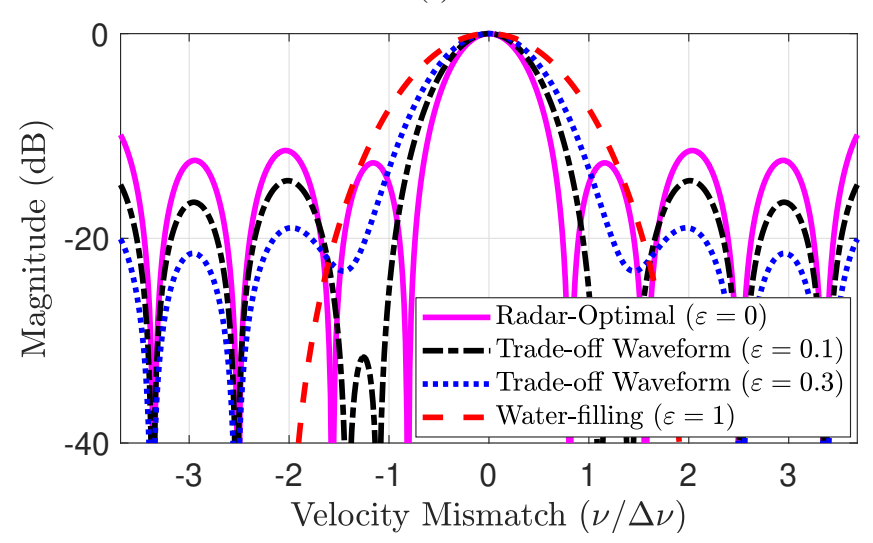

(b)

Fig. 11. (a) Range and (b) velocity profiles of the radar-optimal, DFRC tradeoff and water-filling waveforms at SNR $=0 \mathrm{~dB}$, where the ISL constrain of the radar-optimal waveform is $\beta_{\mathrm{ISL}}=-15 \mathrm{~dB}$, and range and velocity mismatch values are shown in terms of multiples of delay resolution $\Delta \tau$ and Doppler resolution $\Delta \nu$, respectively.

$\varepsilon$, corresponding to specific points along the $\mathrm{SNR}=0 \mathrm{~dB}$ trade-off curve. We observe that as the proximity to the radaroptimal waveform increases (i.e., decreasing $\varepsilon$ ), the curvature of the main-lobe becomes sharper, which indicates improved quality of range and velocity estimates. Evidently, the waterfilling waveform yields the worst estimation performance due to its wide main-lobe.

\section{CONCLUDING REMARKS}

We have addressed the time-frequency waveform design problem for an OFDM DFRC system in the presence of a lowrate feedforward channel between the DFRC transmitter and the communications receiver. The proposed radar-optimal and
DFRC trade-off waveform design algorithms have been shown to provide significant performance gains over the conventional schemes, in terms of both radar estimation accuracy and channel capacity. Moreover, we provide important insights into the structure of optimal waveforms under different SNR and radar similarity regimes. As a future research direction, we plan to extend the proposed design strategy to massive MIMO radarcommunications scenarios, where a massive MIMO BS serves multiple UEs while simultaneously detecting targets using the backscattered signals [82]. In this setup, conveying the information on waveforms and beamforming vectors to UEs might require a huge overhead, which makes it necessary to consider limited feedforward waveform/beamforming designs.

\section{REFERENCES}

[1] F. Liu, C. Masouros, A. Petropulu, H. Griffiths, and L. Hanzo, "Joint radar and communication design: Applications, state-of-the-art, and the road ahead," IEEE Transactions on Communications, pp. 1-1, 2020.

[2] K. V. Mishra, M. R. Bhavani Shankar, V. Koivunen, B. Ottersten, and S. A. Vorobyov, "Toward millimeter-wave joint radar communications: A signal processing perspective," IEEE Signal Processing Magazine, vol. 36, no. 5, pp. 100-114, Sep. 2019.

[3] L. Zheng, M. Lops, Y. C. Eldar, and X. Wang, "Radar and communication coexistence: An overview: A review of recent methods," IEEE Signal Processing Magazine, vol. 36, no. 5, pp. 85-99, 2019.

[4] A. R. Chiriyath, B. Paul, and D. W. Bliss, "Radar-communications convergence: Coexistence, cooperation, and co-design," IEEE Transactions on Cognitive Communications and Networking, vol. 3, no. 1, pp. 1-12, 2017.

[5] C. Sturm and W. Wiesbeck, "Waveform design and signal processing aspects for fusion of wireless communications and radar sensing," Proceedings of the IEEE, vol. 99, no. 7, pp. 1236-1259, July 2011.

[6] C. Aydogdu, M. F. Keskin, G. K. Carvajal, O. Eriksson, H. Hellsten, H. Herbertsson, E. Nilsson, M. Rydstrom, K. Vanas, and H. Wymeersch, "Radar interference mitigation for automated driving: Exploring proactive strategies," IEEE Signal Processing Magazine, vol. 37, no. 4, pp. 72-84, 2020.

[7] A. Hassanien, M. G. Amin, E. Aboutanios, and B. Himed, "Dualfunction radar communication systems: A solution to the spectrum congestion problem," IEEE Signal Processing Magazine, vol. 36, no. 5, pp. $115-126$, Sep. 2019.

[8] F. Liu, L. Zhou, C. Masouros, A. Li, W. Luo, and A. Petropulu, "Toward dual-functional radar-communication systems: Optimal waveform design," IEEE Transactions on Signal Processing, vol. 66, no. 16, pp. 4264-4279, Aug 2018

[9] D. Ma, N. Shlezinger, T. Huang, Y. Liu, and Y. C. Eldar, "Joint radarcommunication strategies for autonomous vehicles: Combining two key automotive technologies," IEEE Signal Processing Magazine, vol. 37, no. 4, pp. 85-97, 2020.

[10] P. Kumari, S. A. Vorobyov, and R. W. Heath, "Adaptive virtual waveform design for millimeter-wave joint communication-radar," IEEE Transactions on Signal Processing, vol. 68, pp. 715-730, 2020.

[11] L. Reichardt, C. Sturm, F. Grünhaupt, and T. Zwick, "Demonstrating the use of the IEEE $802.11 \mathrm{p}$ car-to-car communication standard for automotive radar," in 2012 6th European Conference on Antennas and Propagation (EUCAP), March 2012, pp. 1576-1580.

[12] P. Kumari, J. Choi, N. González-Prelcic, and R. W. Heath, "IEEE 802.11ad-based radar: An approach to joint vehicular communicationradar system," IEEE Transactions on Vehicular Technology, vol. 67, no. 4, pp. 3012-3027, April 2018.

[13] S. H. Dokhanchi, M. R. Bhavani Shankar, K. V. Mishra, and B. Ottersten, "Multi-constraint spectral co-design for colocated MIMO radar and MIMO communications," in ICASSP 2020 - 2020 IEEE International Conference on Acoustics, Speech and Signal Processing (ICASSP), 2020, pp. 4567-4571.

[14] S. H. Dokhanchi, B. S. Mysore, K. V. Mishra, and B. Ottersten, "A mWave automotive joint radar-communications system," IEEE Transactions on Aerospace and Electronic Systems, vol. 55, no. 3, pp. 12411260, 2019.

[15] R. F. Tigrek, W. J. A. De Heij, and P. Van Genderen, "OFDM signals as the radar waveform to solve Doppler ambiguity," IEEE Transactions on Aerospace and Electronic Systems, vol. 48, no. 1, pp. 130-143, Jan 2012.

[16] M. Braun, "OFDM radar algorithms in mobile communication networks," Karlsruher Institutes für Technologie, 2014.

[17] M. Bică and V. Koivunen, "Generalized multicarrier radar: Models and performance," IEEE Transactions on Signal Processing, vol. 64, no. 17, pp. 4389-4402, Sep. 2016. 
[18] Y. Liu, G. Liao, J. Xu, Z. Yang, and Y. Zhang, "Adaptive OFDM integrated radar and communications waveform design based on information theory," IEEE Communications Letters, vol. 21, no. 10, pp. 2174-2177, 2017.

[19] A. Turlapaty, Y. Jin, and Y. Xu, "Range and velocity estimation of radar targets by weighted OFDM modulation," in 2014 IEEE Radar Conference, 2014, pp. 1358-1362.

[20] D. Lim, S. Heo, and J. No, "An overview of peak-to-average power ratio reduction schemes for OFDM signals," Journal of Communications and Networks, vol. 11, no. 3, pp. 229-239, 2009.

[21] T. Huang and T. Zhao, "Low PMEPR OFDM radar waveform design using the iterative least squares algorithm," IEEE Signal Processing Letters, vol. 22, no. 11, pp. 1975-1979, 2015.

[22] R. F. Tigrek, "A processing technique for OFDM-modulated wideband radar signals," 2010.

[23] Y. Liu, G. Liao, Z. Yang, and J. Xu, "Multiobjective optimal waveform design for OFDM integrated radar and communication systems," Signal Processing, vol. 141, pp. 331-342, 2017

[24] M. Bică and V. Koivunen, "Multicarrier radar-communications waveform design for RF convergence and coexistence," in ICASSP 2019-2019 IEEE International Conference on Acoustics, Speech and Signal Processing (ICASSP). IEEE, 2019, pp. 7780-7784.

[25] C. Shi, F. Wang, S. Salous, and J. Zhou, "Joint subcarrier assignment and power allocation strategy for integrated radar and communications system based on power minimization," IEEE Sensors Journal, vol. 19 no. 23, pp. 11 167-11 179, 2019.

[26] Z. Zhang, Z. Du, and W. Yu, "Mutual-information-based OFDM waveform design for integrated radar-communication system in Gaussian mixture clutter," IEEE Sensors Letters, vol. 4, no. 1, pp. 1-4, 2020.

[27] A. Ahmed, Y. D. Zhang, A. Hassanien, and B. Himed, "OFDM-based joint radar-communication system: Optimal sub-carrier allocation and power distribution by exploiting mutual information," in $201953 \mathrm{rd}$ Asilomar Conference on Signals, Systems, and Computers. IEEE, 2019, pp. 559-563.

[28] S. Sen and A. Nehorai, "Adaptive design of OFDM radar signal with improved wideband ambiguity function," IEEE Transactions on Signal Processing, vol. 58, no. 2, pp. 928-933, 2009.

[29] _ "Adaptive OFDM radar for target detection in multipath scenarios," IEEE Transactions on Signal Processing, vol. 59, no. 1, pp. 78-90, 2010.

[30] L. Gaudio, M. Kobayashi, B. Bissinger, and G. Caire, "Performance analysis of joint radar and communication using OFDM and OTFS,' arXiv e-prints, p. arXiv:1902.01184, Feb 2019.

[31] S. Mercier, S. Bidon, D. Roque, and C. Enderli, "Comparison of correlation-based OFDM radar receivers," IEEE Transactions on Aerospace and Electronic Systems, 2020.

[32] C.-B. Chae, D. Mazzarese, T. Inoue, and R. W. Heath, "Coordinated beamforming for the multiuser MIMO broadcast channel with limited feedforward," IEEE Transactions on Signal Processing, vol. 56, no. 12, pp. 6044-6056, 2008.

[33] R. Moosavi, J. Eriksson, E. G. Larsson, N. Wiberg, P. Frenger, and F. Gunnarsson, "Comparison of strategies for signaling of scheduling assignments in wireless OFDMA," IEEE Transactions on Vehicular Technology, vol. 59, no. 9, pp. 4527-4542, Nov 2010.

[34] L. Soriano-Equigua, J. Sanchez-Garcia, J. Flores-Troncoso, and R. W. Heath, "Noniterative coordinated beamforming for multiuser MIMO systems with limited feedforward," IEEE Signal Processing Letters, vol. 18 , no. 12 , pp. 701-704, 2011.

[35] T. Lipp and S. Boyd, "Variations and extension of the convex-concave procedure," Optimization and Engineering, vol. 17, no. 2, pp. 263-287, 2016 .

[36] K. Shen and W. Yu, "Fractional programming for communication systems - part i: Power control and beamforming," IEEE Transactions on Signal Processing, vol. 66, no. 10, pp. 2616-2630, 2018.

[37] P. Stoica, Y. Jiang, and J. Li, "On MIMO channel capacity: an intuitive discussion," IEEE Signal Processing Magazine, vol. 22, no. 3, pp. 83-84, May 2005 .

[38] J. P. González-Coma, A. Gründinger, M. Joham, and L. Castedo, "MSE balancing in the MIMO BC: Unequal targets and probabilistic interference constraints," IEEE Transactions on Signal Processing, vol. 65 no. 12, pp. 3293-3305, June 2017.

[39] M. Biedka, Y. E. Wang, Q. M. Xu, and Y. Li, "Full-duplex RF front ends : From antennas and circulators to leakage cancellation," IEEE Microwave Magazine, vol. 20, no. 2, pp. 44-55, 2019.

[40] C. B. Barneto, T. Riihonen, M. Turunen, L. Anttila, M. Fleischer, K. Stadius, J. Ryynänen, and M. Valkama, "Full-duplex OFDM radar with LTE and 5G NR waveforms: Challenges, solutions, and measurements," IEEE Transactions on Microwave Theory and Techniques, vol. 67, no. 10, pp. 4042-4054, 2019.

[41] C. R. Berger, B. Demissie, J. Heckenbach, P. Willett, and S. Zhou, "Signal processing for passive radar using OFDM waveforms," IEEE Journal of Selected Topics in Signal Processing, vol. 4, no. 1, pp. 226$238,2010$.

[42] L. Zheng and X. Wang, "Super-resolution delay-Doppler estimation for OFDM passive radar," IEEE Transactions on Signal Processing, vol. 65 no. 9, pp. 2197-2210, May 2017.
[43] A. Goldsmith, Wireless Communications. Cambridge University Press, 2005.

[44] L. Zheng, M. Lops, X. Wang, and E. Grossi, "Joint design of overlaid communication systems and pulsed radars," IEEE Transactions on Signal Processing, vol. 66, no. 1, pp. 139-154, Jan 2018.

[45] T.-H. Chang, W.-K. Ma, and C.-Y. Chi, "Maximum-likelihood detection of orthogonal space-time block coded OFDM in unknown block fading channels," IEEE Transactions on Signal Processing, vol. 56, no. 4, pp. 1637-1649, 2008

[46] S. Ohno and G. B. Giannakis, "Capacity maximizing MMSE-optimal pilots for wireless OFDM over frequency-selective block Rayleighfading channels," IEEE Transactions on Information Theory, vol. 50, no. 9, pp. 2138-2145, Sep. 2004.

[47] S. Ye, S. H. Wong, and C. Worrall, "Enhanced physical downlink control channel in LTE advanced release 11," IEEE Communications Magazine, vol. 51, no. 2, pp. 82-89, February 2013.

[48] H. L. V. Trees, Detection, Estimation, and Modulation Theory. John Wiley \& Sons, New York, 2004.

[49] Z. Shen, J. G. Andrews, and B. L. Evans, "Adaptive resource allocation in multiuser OFDM systems with proportional rate constraints," IEEE Transactions on Wireless Communications, vol. 4, no. 6, pp. 2726-2737, 2005.

[50] A. Assalini, E. Dall'Anese, and S. Pupolin, "On the robustness of MIMO LMMSE channel estimation," IEEE Transactions on Wireless Communications, vol. 9, no. 11, pp. 3313-3319, 2010.

[51] S. M. Kay, Fundamentals of Statistical Signal Processing: Estimation Theory. Prentice Hall, 1993.

[52] M. Ding and S. D. Blostein, "Maximum mutual information design for MIMO systems with imperfect channel knowledge," IEEE transactions on Information Theory, vol. 56, no. 10, pp. 4793-4801, 2010.

[53] Y. Polyanskiy, H. V. Poor, and S. Verdu, "Channel coding rate in the finite blocklength regime," IEEE Transactions on Information Theory, vol. 56, no. 5, pp. 2307-2359, 2010.

[54] J. Jang and K. B. Lee, "Transmit power adaptation for multiuser OFDM systems," IEEE Journal on Selected Areas in Communications, vol. 21, no. 2, pp. 171-178, 2003.

[55] Y. Yao and G. B. Giannakis, "Rate-maximizing power allocation in OFDM based on partial channel knowledge," IEEE Transactions on Wireless Communications, vol. 4, no. 3, pp. 1073-1083, May 2005.

[56] H. Q. Ngo, E. G. Larsson, and T. L. Marzetta, "Energy and spectral efficiency of very large multiuser MIMO systems," IEEE Transactions on Communications, vol. 61, no. 4, pp. 1436-1449, 2013.

[57] E. Björnson, E. G. Larsson, and M. Debbah, "Massive MIMO for maximal spectral efficiency: How many users and pilots should be allocated?" IEEE Transactions on Wireless Communications, vol. 15, no. 2, pp. 1293-1308, 2016.

[58] J. Qian, M. Lops, , X. Wang, and Z. He, "Joint system design for coexistence of MIMO radar and MIMO communication," IEEE Transactions on Signal Processing, vol. 66, no. 13, pp. 3504-3519, July 2018.

[59] E. Grossi, M. Lops, and L. Venturino, "Joint design of surveillance radar and MIMO communication in cluttered environments," IEEE Transactions on Signal Processing, vol. 68, pp. 1544-1557, 2020.

[60] M. Bică and V. Koivunen, "Radar waveform optimization for target parameter estimation in cooperative radar-communications systems," IEEE Transactions on Aerospace and Electronic Systems, vol. 55, no. 5, pp. 2314-2326, Oct 2019

[61] S. P. Sira, Y. Li, A. Papandreou-Suppappola, D. Morrell, D. Cochran, and M. Rangaswamy, "Waveform-agile sensing for tracking," IEEE Signal Processing Magazine, vol. 26, no. 1, pp. 53-64, Jan 2009.

[62] J. Zhang, H. Wang, and X. Zhu, "Adaptive waveform design for separated transmit/receive ULA-MIMO radar," IEEE Transactions on Signal Processing, vol. 58, no. 9, pp. 4936-4942, Sep. 2010.

[63] C. Gao, K. C. Teh, and A. Liu, "Piecewise nonlinear frequency modulation waveform for MIMO radar," IEEE Journal of Selected Topics in Signal Processing, vol. 11, no. 2, pp. 379-390, March 2017.

[64] M. A. Richards, Fundamentals of Radar Signal Processing. Tata McGraw-Hill Education, 2005.

[65] F. Alizadeh and D. Goldfarb, "Second-order cone programming," Mathematical Programming, vol. 95, no. 1, pp. 3-51, 2003.

[66] B. K. Sriperumbudur and G. R. Lanckriet, "On the convergence of the concave-convex procedure," in Proceedings of the 22nd International Conference on Neural Information Processing Systems. Curran Associates Inc., 2009, pp. 1759-1767.

[67] Z. Yang, L. Xie, and P. Stoica, "Vandermonde decomposition of multilevel Toeplitz matrices with application to multidimensional superresolution," IEEE Transactions on Information Theory, vol. 62, no. 6, pp. 3685-3701, June 2016

[68] M. Grant and S. Boyd, "CVX: Matlab software for disciplined convex programming, version 2.1," http://cvxr.com/cvx, Mar. 2014

[69] R. Moosavi and E. G. Larsson, "Reducing physical layer control signaling using mobile-assisted scheduling," IEEE transactions on wireless communications, vol. 12, no. 1, pp. 368-379, 2012.

[70] A. Aubry, A. De Maio, A. Zappone, M. Razaviyayn, and Z.-Q. Luo, "A new sequential optimization procedure and its applications to resource 
allocation for wireless systems," IEEE Transactions on Signal Processing, vol. 66, no. 24, pp. 6518-6533, 2018.

[71] S. Boyd and L. Vandenberghe, Convex Optimization. Cambridge university press, 2004.

[72] W. Huyer and A. Neumaier, "Global optimization by multilevel coordinate search," Journal of Global Optimization, vol. 14, no. 4, pp. 331-355, 1999.

[73] J. M. Geist, "Capacity and cutoff rate for dense M-ary PSK constellations," in IEEE Conference on Military Communications. IEEE, 1990, pp. $768-770$.

[74] B. Paul and D. W. Bliss, "Extending joint radar-communications bounds for FMCW radar with Doppler estimation," in IEEE Radar Conference (RadarCon). IEEE, 2015, pp. 0089-0094.

[75] B. Paul, A. R. Chiriyath, and D. W. Bliss, "Joint communications and radar performance bounds under continuous waveform optimization: The waveform awakens," in 2016 IEEE Radar Conference (RadarConf). IEEE, 2016, pp. 1-6.

[76] P. Stoica, R. L. Moses et al., Spectral Analysis of Signals. Pearson Prentice Hall Upper Saddle River, NJ, 2005.

[77] H. Wymeersch, G. Seco-Granados, G. Destino, D. Dardari, and F. Tufvesson, "5G mmWave positioning for vehicular networks," IEEE Wireless Communications, vol. 24, no. 6, pp. 80-86, 2017.

[78] H. V. Cheng, D. Persson, and E. G. Larsson, "Optimal MIMO precoding under a constraint on the amplifier power consumption," IEEE Transactions on Communications, vol. 67, no. 1, pp. 218-229, 2018.

[79] H. Moon, "Waterfilling power allocation at high SNR regimes," IEEE Transactions on Communications, vol. 59, no. 3, pp. 708-715, 2011.

[80] P. Viswanath, D. N. C. Tse, and V. Anantharam, "Asymptotically optimal water-filling in vector multiple-access channels," IEEE Transactions on Information Theory, vol. 47, no. 1, pp. 241-267, 2001.

[81] D. Tse and P. Viswanath, Fundamentals of Wireless Communication. Cambridge university press, 2005.

[82] F. Liu, C. Masouros, A. Li, H. Sun, and L. Hanzo, "MU-MIMO communications with MIMO radar: From co-existence to joint transmission,' IEEE Transactions on Wireless Communications, vol. 17, no. 4, pp. 2755-2770, 2018. 